\title{
Self dual, reversible and complementary duals constacyclic codes over finite local Frobenius non-chain rings of length 5 and nilpotency index 4 .
}

\author{
C. A. Castillo-Guillén and C. Álvarez-García
}

\begin{abstract}
Over finite local Frobenius non-chain rings of length 5 and nilpotency index 4 and when the length of the code is relatively prime to the characteristic of the residue field of the ring, the structure of the dual of $\gamma$-constacyclic codes is established and the algebraic characterization of self-dual, reversible $\gamma$-constacyclic codes and $\gamma$-constacyclic codes with complementary dual are given.
\end{abstract}

\section{Introduction}

After the work of R. Hammons et al. see [7] codes over finite rings have received considerable attention. Parallel to this, constacyclic codes, self dual codes, reversible codes and codes with complementary duals over finite rings have also gained a large popularity among coding theorists. For some of the works in these directions we refer to [1] [2], [3], [4], [6], [8], [9], [14], [17]. The class of $\gamma$-constacyclic codes plays a very significant role in the theory of errorcorrecting codes, these are codes invariant under the mapping $\sigma_{\gamma}: \mathrm{R}^{n} \longrightarrow \mathrm{R}^{n}$ given by $\sigma_{\gamma}\left(a_{0}, \ldots, a_{n-1}\right)=\left(\gamma a_{n-1}, a_{0}, \ldots, a_{n-2}\right), \gamma$ is a unit of the ring R. Reversible codes are codes invariant under the mapping $\mathfrak{r}: \mathrm{R}^{n} \longrightarrow \mathrm{R}^{n}$ given

Key Words: Complementary dual codes, Constacyclic codes, Dual codes, Frobenius rings, Reversible codes, Self-dual codes.

2010 Mathematics Subject Classification: Primary 94B15; Secondary $94 B 05$.

Received: 29.08.2020

Accepted: 30.10 .2020 
SELF DUAL, REVERSIBLE AND COMPLEMENTARY DUALS

CONSTACYCLIC CODES OVER FINITE LOCAL FROBENIUS NON-CHAIN RINGS OF LENGTH 5 AND NILPOTENCY INDEX 4

by $\mathfrak{r}\left(a_{0}, \ldots, a_{n-1}\right)=\left(a_{n-1}, \ldots, a_{0}\right)$, see [10]. These codes are used to obtain DNA codes. Linear complementary dual codes (which is abbreviated to LCD code) are linear codes that trivially intersect with their duals, [11]. Over fields cyclic LCD codes are the reversible codes and LCD codes have a good asymptotically property and meet the asymptotic Gilbert-Varshamov bound, see $[13],[16]$.

Duals of $\gamma$-constacyclic codes, self dual, reversible $\gamma$-constacyclic codes and $\gamma$-constacyclic codes with complementary duals over finite local Frobenius non-chain rings whose maximal ideal has nilpotency index 3 and the length of the code is relatively prime to the characteristic of the residue field of the ring were studied in [3], [4]. In [5], finite local Frobenius non-chain rings of length 5 whose maximal ideal has nilpotency index 4 were determined and the structure of $\gamma$-constacyclic codes over these rings was described. Now, it would be interesting to determine the dual of $\gamma$-constacyclic codes and the algebraic characterization of self-dual, reversible $\gamma$-constacyclic codes and $\gamma$-constacyclic codes with complementary duals over finite local Frobenius non-chain rings of length 5 whose maximal ideal has nilpotency index 4 .

Throughout this work all codes are over finite local Frobenius non-chain rings of length 5 whose maximal ideal has nilpotency index 4 and the length of the code is relatively prime to the characteristic of the residue field of the ring. The purpose of this paper is to obtain structure Theorems for the dual of a constacyclic code, for self-dual constacyclic codes, for reversible constacyclic codes and for constacyclic codes with complementary duals. The paper is organized as follows: in Section 2 facts on finite commutative local rings are recalled, properties of the reciprocal polynomial over local rings and properties of finite local Frobenius non-chain rings of length 5 whose maximal ideal has nilpotency index 4 are given. In Section 3 the structure of the dual of a $\gamma$ constacyclic code is determined, generators for the dual code are obtained from the generators of the original constacyclic code. The algebraic characterization of self-dual $\gamma$-constacyclic codes is presented in Section 4. In Section 5 the algebraic characterization of reversible $\gamma$-constacyclic codes is presented. The algebraic characterization of complementary $\gamma$-constacyclic codes is given in Section 6. Examples are included to illustrate the main results.

\section{Preliminaries}

Throughout this work $\operatorname{GF}(q)$ denotes the finite field with $q=p^{d}$ elements, $p$ a prime, all rings are assumed to be finite, commutative with unit element and all modules are finitely generated. 
SELF DUAL, REVERSIBLE AND COMPLEMENTARY DUALS CONSTACYCLIC CODES OVER FINITE LOCAL FROBENIUS NON-CHAIN RINGS OF LENGTH 5 AND NILPOTENCY INDEX 4

\subsection{Rings and local rings}

Let $\mathrm{R}$ and $\mathrm{B}$ be rings, $\mathrm{I}$ an ideal of $\mathrm{R}$ and $\mathrm{M}$ an $\mathrm{R}$-module. The length of $\mathrm{M}$, denoted by $\ell_{\mathrm{R}}(\mathrm{M})$, is the length of a composition series for $\mathrm{M}$. The annihilator ideal of $\mathrm{M}$ in $\mathrm{R}$ is defined as $\operatorname{ann}_{\mathrm{R}}(\mathrm{M}):=\{a \in \mathrm{R}: a m=0, \forall m \in \mathrm{M}\}$. If $\mathrm{I}$ is contained in $\operatorname{ann}_{R}(\mathrm{M})$ there is a natural structure of $(\mathrm{R} / \mathrm{I})$-module on $\mathrm{M}$, the scalar multiplication is given by $(a+\mathrm{I}, m) \mapsto a m, a \in \mathrm{R}, m \in \mathrm{M}$, and the lattice of R-submodules and (R/I)-submodules of $\mathrm{M}$ are the same. If $\mathrm{R}$ has only one maximal ideal $\mathfrak{m}$ then it is called local, $\mathrm{k}=\mathrm{R} / \mathfrak{m}$ its residue field and it will be denoted by the triple $(\mathrm{R}, \mathfrak{m}, \mathrm{GF}(q))$. $\mathrm{R}$ is called a chain ring if the lattice of its ideals is a chain under set-theoretic inclusion. A finite local ring $(\mathrm{R}, \mathfrak{m}, \mathrm{GF}(q))$ is Frobenius if $\operatorname{ann}_{\mathrm{R}}(\mathfrak{m})$ is the unique minimal ideal of $\mathrm{R}$, see [15]. $\mathfrak{F}_{5}^{4}$ denotes the family of finite local Frobenius non-chain rings of length 5 whose maximal ideal has nilpotency index 4 , see [5].

If $(\mathrm{R}, \mathfrak{m}, \mathrm{GF}(q))$ is a finite local ring, then $|\mathrm{M}|=|\mathrm{GF}(q)|^{\ell_{\mathrm{R}}(\mathrm{M})}$, see [2], and there is an integer $t \geq 1$ such that $\mathfrak{m}^{t}=\langle 0\rangle$ and $\mathfrak{m}^{t-1} \neq\langle 0\rangle$, called the nilpotency index of $\mathfrak{m}$. A subset $\mathrm{G}$ of $\mathrm{M}$ generates $\mathrm{M}$ if and only if its image $\overline{\mathrm{G}}$ in $\mathrm{M} / \mathfrak{m M}$ generates $\mathrm{M} / \mathfrak{m M}$ as a $\mathrm{GF}(q)$-vector space. A set of generators for $\mathrm{M}$ obtained from lifting a basis of the $\mathrm{GF}(q)$-vector space $\mathrm{M} / \mathrm{mM}$ is called a minimal Rgenerating set for $\mathrm{M}$ and $\mathrm{v}_{\mathrm{R}}(\mathrm{M})$ denotes the number of elements in a minimal $\mathrm{R}$-generating set for $\mathrm{M}$, see [12]. Note that $\mathrm{v}_{\mathrm{R}}(\mathrm{M})=\operatorname{dim}_{\mathrm{GF}(q)}(\mathrm{M} / \mathfrak{m M})=$ $\ell_{\mathrm{R}}(\mathrm{M} / \mathrm{mM})$.

Let $(\mathrm{R}, \mathfrak{m}, \mathrm{GF}(q))$ be a finite local ring, $\mathrm{f}, \mathrm{g} \in \mathrm{R}[\mathrm{T}], \gamma$ a unit of $\mathrm{R}$ and $n \in \mathbb{N}$ with $(n, q)=1 .^{-}: \mathrm{R}[\mathrm{T}] \rightarrow \mathrm{GF}(q)[\mathrm{T}]$ is the natural ring homomorphism given by $a_{0}+a_{1} \mathrm{~T}+\ldots+a_{m} \mathrm{~T}^{m} \mapsto\left(a_{0}+\mathfrak{m}\right)+\left(a_{1}+\mathfrak{m}\right) \mathrm{T}+\ldots+\left(a_{m}+\mathfrak{m}\right) \mathrm{T}^{m}$, the polynomial $\mathrm{f} \in \mathrm{R}[\mathrm{T}]$ is called basic irreducible if $\overline{\mathrm{f}}$ is irreducible in $\mathrm{GF}(q)[\mathrm{T}]$, $\mathrm{f}$ and $\mathrm{g}$ are called coprime if $\langle\mathrm{f}\rangle+\langle\mathrm{g}\rangle=\mathrm{R}[\mathrm{T}]$. Hensel's Lemma guarantees that factorization into a product of pairwise coprime polynomials in $\mathrm{GF}(q)[\mathrm{T}]$ lifts to such a factorization over $\mathrm{R}[\mathrm{T}]$, see [12]. In fact there is a unique family of monic basic irreducible pairwise coprime polynomials such that $\mathrm{T}^{n}-\gamma=$ $\mathrm{f}_{1} \cdots \mathrm{f}_{r}$, see [3].

Let $\mathrm{f} \in \mathrm{R}[\mathrm{T}]$ be a monic basic irreducible polynomial, $\operatorname{deg}(\overline{\mathrm{f}})=s, \mathbb{T} \subset \mathrm{R}$ be a set of representatives of $\operatorname{GF}(q)$ and I an ideal of R. From [2], [3] and [5] we have: (1) $\mathrm{B}=\mathrm{R}[\mathrm{T}] /\langle\mathrm{f}\rangle=\left\{a_{0}+a_{1} \mathrm{~T}+\cdots+a_{s-1} \mathrm{~T}^{s-1}: a_{i} \in \mathrm{R}\right\}$ is a local separable extension of $\mathrm{R}$ with maximal ideal $\mathfrak{m B}$ and residue field $\operatorname{GF}\left(q^{s}\right)$; (2) the set $\mathbb{T}_{s}:=\left\{a_{0}+a_{1} \mathrm{~T}+\cdots+a_{s-1} \mathrm{~T}^{s-1}: a_{i} \in \mathbb{T}\right\} \subset \mathrm{B}$ is a set of representatives of $\mathrm{GF}\left(q^{s}\right)$; (3) the nilpotency index of $\mathfrak{m}$ and $\mathfrak{m B}$ is the same; (4) $\ell_{\mathrm{R}}(\mathrm{I})=\ell_{\mathrm{B}}(\mathrm{IB}) ;(5) \mathrm{v}_{\mathrm{R}}(\mathrm{I})=\mathrm{v}_{\mathrm{B}}(\mathrm{IB}) ;(6) \mathrm{A}$ minimal R-generating set for I is a minimal B-generating set for IB; $(7)\left(\operatorname{ann}_{R}(\mathrm{I})\right) \mathrm{B}=\operatorname{ann}_{\mathrm{B}}(\mathrm{IB}) ;(8) \mathrm{R}$ is a chain ring if and only if $B$ is a chain ring; (9) $R$ is Frobenius if and only if $B$ is Frobenius, in this case $\operatorname{ann}_{\mathrm{B}}(\mathfrak{m B})=\mathfrak{m}^{t-1} \mathrm{~B}$, where $t$ is the nilpotency index 
SELF DUAL, REVERSIBLE AND COMPLEMENTARY DUALS

CONSTACYCLIC CODES OVER FINITE LOCAL FROBENIUS NON-CHAIN

RINGS OF LENGTH 5 AND NILPOTENCY INDEX 4

of $\mathfrak{m}$.

If $\mathrm{R}$ is a finite local Frobenius ring, (10) $\operatorname{ann}_{\mathrm{R}}\left(\operatorname{ann}_{\mathrm{R}}(\mathrm{I})\right)=\mathrm{I} ;(11) \ell_{\mathrm{R}}\left(\operatorname{ann}_{\mathrm{R}}(\mathrm{I})\right)+$ $\ell_{\mathrm{R}}(\mathrm{I})=\ell_{\mathrm{R}}(\mathrm{R}) ;(12) \mathfrak{m}^{i-1} \operatorname{ann}_{\mathrm{R}}\left(\mathfrak{m}^{i}\right)=\mathfrak{m}^{t-1}, 2 \leq i \leq t-1 ;(13) \mathrm{v}_{\mathrm{R}}\left(\operatorname{ann}_{\mathrm{R}}\left(\mathfrak{m}^{2}\right)\right)=$ $\mathrm{v}_{\mathrm{R}}(\mathfrak{m}) ; \quad(14)(\mathfrak{m B})^{i-1} \operatorname{ann}_{\mathrm{B}}\left((\mathfrak{m B})^{i}\right)=\mathfrak{m}^{i-1} \operatorname{ann}_{\mathrm{B}}\left(\mathfrak{m}^{i} \mathrm{~B}\right)=\mathfrak{m}^{i-1} \operatorname{ann}_{\mathrm{R}}\left(\mathfrak{m}^{i}\right) \mathrm{B}=$ $\mathfrak{m}^{t-1} \mathrm{~B}=(\mathfrak{m B})^{t-1}, 2 \leq i \leq t-1 ;(15) \mathrm{v}_{\mathrm{B}}(\mathfrak{m B})=\mathrm{v}_{\mathrm{R}}(\mathfrak{m})=\mathrm{v}_{\mathrm{R}}\left(\operatorname{ann}_{\mathrm{R}}\left(\mathfrak{m}^{2}\right)\right)=$ $\mathrm{v}_{\mathrm{B}}\left(\operatorname{ann}_{\mathrm{R}}\left(\mathfrak{m}^{2}\right) \mathrm{B}\right)=\mathrm{v}_{\mathrm{B}}\left(\operatorname{ann}_{\mathrm{B}}\left(\mathfrak{m}^{2} \mathrm{~B}\right)\right)=\mathrm{v}_{\mathrm{B}}\left(\operatorname{ann}_{\mathrm{B}}\left((\mathfrak{m B})^{2}\right)\right)$.

Let $(\mathrm{R}, \mathfrak{m}, \mathrm{GF}(q))$ be a finite local Frobenius ring, $\mathrm{f} \in \mathrm{R}[\mathrm{T}]$ be a basic irreducible polynomial, $s=\operatorname{deg}(\overline{\mathrm{f}}),\left(\mathrm{B}=\mathrm{R}[\mathrm{T}] /\langle\mathrm{f}\rangle, \mathfrak{m B}, \mathrm{GF}\left(q^{s}\right)\right)$ the separable extension of $\mathrm{R}$ determined by $\mathrm{f}, \mathbb{T} \subset \mathrm{R}$ a set of representatives of $\mathrm{GF}(q)$ with $0,1 \in \mathbb{T}, \mathrm{M}$ a B-module, $\tilde{\theta}=\left\{\theta_{1}, \ldots, \theta_{l}\right\}$ a sequence of elements of $\mathrm{M}$ and $\mathrm{H}=\left(h_{i j}\right)$ a $(k \times l)$ matrix over $\operatorname{GF}\left(q^{s}\right)$. For the remainder of this work the following notation will be used:

(1) The number $\mathrm{v}_{\mathrm{R}}(\mathfrak{m})=\mathrm{v}_{\mathrm{R}}\left(\operatorname{ann}_{\mathrm{R}}\left(\mathfrak{m}^{2}\right)\right)=\mathrm{v}_{\mathrm{B}}(\mathfrak{m B})=\mathrm{v}_{\mathrm{B}}\left(\operatorname{ann}_{\mathrm{B}}\left((\mathfrak{m B})^{2}\right)\right)$ is denoted by $\chi$;

(2) $\tilde{\alpha}=\left\{\alpha_{1}, \ldots, \alpha_{\chi}\right\}$ will be a minimal R-generating set for $\mathfrak{m}$;

(3) $\tilde{\beta}=\left\{\beta_{1}, \ldots, \beta_{\chi}\right\}$ will be a minimal R-generating set for $\operatorname{ann}_{\mathrm{R}}\left(\mathfrak{m}^{2}\right)$;

(4) $\mathbb{T}_{s}=\left\{a_{0}+a_{1} \mathrm{~T}+\cdots+a_{s-1} \mathrm{~T}^{s-1}: a_{i} \in \mathbb{T}\right\} \subset \mathrm{B}$ will be the set of representatives of $\mathrm{B} / \mathfrak{m B}=\operatorname{GF}\left(q^{s}\right)$;

(5) For $a \in \operatorname{GF}\left(q^{s}\right), a^{\mathbb{T}_{s}}$ will denote the unique representative of $a$ in $\mathbb{T}_{s}$;

(6) The B-submodule of $\mathrm{M},\left\langle h_{11}^{\mathbb{T}_{s}} \theta_{1}+\ldots+h_{1 l}^{\mathbb{T}_{s}} \theta_{l}, \ldots, h_{k 1}^{\mathbb{T}_{s}} \theta_{1}+\ldots+h_{k l}^{\mathbb{T}_{s}} \theta_{l}\right\rangle$, will be denoted by $\mathrm{H}_{\tilde{\theta}}^{\mathbb{T}} s$.

A $(k \times n)$ matrix over $\operatorname{GF}(q)$ is said to be in reduced row echelon form, (rre)-form, if in each row, $i=1, \ldots, k$, the first nonzero entry is equal to 1 , the index of the column in which the 1 occurs, called a pivotal column, strictly increases with $i$, and the $k$ pivotal columns are, in order, the columns of the $(k \times k)$ identity matrix. Since each matrix is row equivalent to a unique reduced row echelon form matrix, the $k$-dimensional vector subspaces of $\mathrm{V}$ are in one-to-one correspondence with the $(k \times n)$ matrices over $\mathrm{GF}(q)$ in $($ rre $)$ form. The $(k \times n)$ matrix $\left(a_{i j}\right)$ over the field in $(r r e)$-form corresponds to the subspace $\left\langle\sum_{i=1}^{n} a_{1 i} \alpha_{i}, \ldots, \sum_{i=1}^{n} a_{k i} \alpha_{i}\right\rangle$.

Lemma 1. ([2], Lemma 1) Let $(\mathrm{R}, \mathfrak{m}, \mathrm{GF}(q))$ be a finite local ring, $\mathbb{T} \subseteq \mathrm{R}$ a set of representatives of $\mathrm{GF}(q), \mathrm{M}$ an $\mathrm{R}$-module and $\tilde{\theta}=\left\{\theta_{1}, \ldots, \theta_{l}\right\}$ be a minimal $\mathrm{R}$-generating set for $\mathrm{M}$. Then the $\mathrm{B}$-submodules of $\mathrm{M}$ between $\mathrm{M}$ and $\mathrm{m} \mathrm{M}$ of length $k+\ell_{\mathrm{R}}(\mathfrak{m M})$, where $0<k<l=\operatorname{dim}_{\mathrm{GF}(q)}(\mathrm{M} / \mathfrak{m M})=\mathrm{v}_{\mathrm{R}}(\mathrm{M})$, are in one to one correspondence with the $(k \times l)$ matrices over $\mathrm{GF}(q)$ in (rre)-form. The matrix $\mathrm{H}=\left(\overline{a_{i j}}\right)$ corresponds to the submodule $\left\langle\sum_{i=1}^{n} a_{1 i} \theta_{i}, \ldots, \sum_{i=1}^{n} a_{k i} \theta_{i}\right\rangle+$ $\mathfrak{m M}$. 
SELF DUAL, REVERSIBLE AND COMPLEMENTARY DUALS

Observation 1. Let $(\mathrm{R}, \mathfrak{m}, \mathrm{GF}(q))$ be a finite local Frobenius ring, $\mathrm{f}, \mathrm{s}$ and $\left(\mathrm{B}, \mathfrak{m B}, \mathrm{GF}\left(q^{s}\right)\right)$ be as above. Let $\mathfrak{F}=\left\{\mathrm{J}\right.$ ideal of $\mathrm{B}: \operatorname{ann}_{\mathrm{B}}(\mathfrak{m B}) \subset \mathrm{J} \subset$ $\left.\operatorname{ann}_{\mathrm{B}}\left((\mathfrak{m B})^{2}\right)\right\}, \mathrm{J} \in \mathfrak{F}, \mathfrak{G}=\left\{\mathrm{I}\right.$ ideal of $\left.\mathrm{B}:(\mathfrak{m B})^{2} \subset \mathrm{I} \subset \mathfrak{m B}\right\}$ and $\mathrm{I} \in \mathfrak{G}$, then:

(1) From relation $\operatorname{ann}_{\mathrm{B}}\left(\operatorname{ann}_{\mathrm{B}}(*)\right)=*$, there is a one to one correspondence between $\mathfrak{F}$ and $\mathfrak{G}$.

(2) $\mathrm{J}$ has length $k+1, k \in\{1, \ldots, \chi-1\}$, and $\operatorname{ann}_{\mathrm{B}}(\mathrm{J})$ has length $\ell_{\mathrm{B}}(\mathrm{B})-$ $k-1=\chi-k+\ell_{\mathrm{R}}\left(\mathfrak{m}^{2}\right)$.

(3) From Lemma 1, relations $\operatorname{ann}_{\mathrm{B}}\left((\mathfrak{m B})^{2}\right)=\operatorname{ann}_{\mathrm{R}}\left(\mathfrak{m}^{2}\right) \mathrm{B}$ and $\operatorname{ann}_{\mathrm{R}}(\mathfrak{m}) \mathrm{B}=$ $\operatorname{ann}_{\mathrm{B}}(\mathfrak{m B})=(\mathfrak{m B}) \operatorname{ann}_{\mathrm{B}}\left((\mathfrak{m B})^{2}\right), \mathrm{J}$ corresponds to a unique $k \times \chi$ matrix in (rre)-form over $\mathrm{GF}\left(q^{s}\right)$ and $\operatorname{ann}_{\mathrm{B}}(\mathrm{J})$ corresponds to a unique $(\chi-$ $k) \times \chi$ matrix in (rre)-form over $\operatorname{GF}\left(q^{s}\right)$.

(4) If $\mathrm{H}_{\mathrm{J}}=\left(a_{i j}\right)$ is the matrix that corresponds to $\mathrm{J}$, the matrix that corresponds to $\operatorname{ann}_{\mathrm{B}}(\mathrm{J})$ will be denoted by $\mathrm{H}_{\mathrm{J}}^{\perp}=\left(b_{i j}\right)$.

Let $\pi \in\{1, \ldots, k\}$ and $\varpi \in\{1, \ldots, \chi-k\}$, we have the following relations:

(a) $\mathrm{J}=\left(\mathrm{H}_{\mathrm{J}}\right)_{\tilde{\beta}}^{\mathbb{T}_{s}}$,

(b) $\operatorname{ann}_{\mathrm{B}}(\mathrm{J})=\left(\mathrm{H}_{\mathrm{J}}^{\perp}\right)_{\tilde{\alpha}}^{\mathbb{T}_{s}}+\mathfrak{m}^{2} \mathrm{~B}$,

(c) $\left(a_{\pi 1}^{\mathbb{T}_{s}} \beta_{1}+\ldots+a_{\pi \chi}^{\mathbb{T}_{s}} \beta_{\chi}\right)\left(b_{\varpi 1}^{\mathbb{T}_{s}} \alpha_{1}+\ldots+b_{\varpi \chi}^{\mathbb{T}_{s}} \alpha_{\chi}\right)=0$.

(5) I has length $k_{1}+\ell_{\mathrm{R}}\left(\mathfrak{m}^{2}\right), k_{1} \in\{1, \ldots, \chi-1\}$, and $\operatorname{ann}_{\mathrm{B}}(\mathrm{I})$ has length $\ell_{\mathrm{B}}(\mathrm{B})-k_{1}-\ell_{\mathrm{R}}\left(\mathfrak{m}^{2}\right)=\chi-k_{1}+1$.

(6) From Lemma 1, I corresponds to a unique $k_{1} \times \chi$ matrix in (rre)-form over $\mathrm{GF}\left(q^{s}\right)$ and $\operatorname{ann}_{\mathrm{B}}(\mathrm{I})$ corresponds to a unique $\left(\chi-k_{1}\right) \times \chi$ matrix in (rre)-form, over $\mathrm{GF}\left(q^{s}\right)$.

(7) If $\mathrm{H}_{\mathrm{I}}=\left(c_{i j}\right)$ is the matrix that corresponds to I, the matrix that corresponds to $\operatorname{ann}_{\mathrm{B}}(\mathrm{I})$ will be denoted by $\mathrm{H}_{\mathrm{I}}^{\perp}=\left(d_{i j}\right)$.

Let $\pi \in\left\{1, \ldots, k_{1}\right\}$ and $\varpi \in\left\{1, \ldots, \chi-k_{1}\right\}$, we have the following relations:

(a) $\mathrm{I}=\left(\mathrm{H}_{\mathrm{I}}\right)_{\tilde{\alpha}}^{\mathbb{T}_{s}}+\mathfrak{m}^{2} \mathrm{~B}$,

(b) $\operatorname{ann}_{\mathrm{B}}(\mathrm{I})=\left(\mathrm{H}_{\mathrm{I}}^{\perp}\right)_{\tilde{\beta}}^{\mathbb{T}_{s}}$,

(c) $\left(c_{\pi 1}^{\mathbb{T}_{s}} \alpha_{1}+\ldots+c_{\pi \chi}^{\mathbb{T}_{s}} \alpha_{\chi}\right)\left(d_{\varpi 1}^{\mathbb{T}_{s}} \beta_{1}+\ldots+d_{\varpi \chi}^{\mathbb{T}_{s}} \beta_{\chi}\right)=0$. 
SELF DUAL, REVERSIBLE AND COMPLEMENTARY DUALS CONSTACYCLIC CODES OVER FINITE LOCAL FROBENIUS NON-CHAIN RINGS OF LENGTH 5 AND NILPOTENCY INDEX 4

\subsection{The reciprocal polynomial and the reciprocal matrix}

Let $(\mathrm{R}, \mathfrak{m}, \mathrm{GF}(q))$ be a finite local ring, $\mathrm{f}, \mathrm{g} \in \mathrm{R}[\mathrm{T}], \gamma$ be a unit of $\mathrm{R}, n \in \mathbb{N}$, with $(n, q)=1$. Let $\mathrm{f}_{1}, \ldots, \mathrm{f}_{r}$ the unique family of monic basic irreducible pairwise coprime polynomials such that $\mathrm{T}^{n}-\gamma=\mathrm{f}_{1} \cdots \mathrm{f}_{r}$. If $a_{0}$ is the constant coefficient of $\mathrm{f}$ and $a_{0}$ is a unit, the polynomial $a_{0}^{-1} \mathrm{f}$ is denoted by $\mathrm{f}^{\circ}$. If $\mathrm{f}$ is a factor of $\mathrm{T}^{n}-\gamma$ or $\mathrm{T}^{n}-\gamma^{-1}$, we will just write $\widehat{\mathrm{f}}=\frac{\mathrm{T}^{n}-\gamma}{\mathrm{f}}$ or $\widehat{\mathrm{f}}=\frac{\mathrm{T}^{n}-\gamma^{-1}}{\mathrm{f}}$, according to the case. The reciprocal of the polynomial $f$ is defined as $\mathrm{f}^{*}=$ $T^{\operatorname{deg}(f)} f\left(\frac{1}{T}\right)$. The polynomial $f$ is called self-reciprocal if $f^{*}$ is an associate of $f$. From [3] and [4] we have the following: (1) $T^{\operatorname{deg}(f)-\operatorname{deg}\left(f^{*}\right)\left(f^{*}\right)^{*}=f ; ~(2) ~ I f ~}$ the constant coefficient of $\mathrm{f}$ is not zero, then $\operatorname{deg}(\mathrm{f})=\operatorname{deg}\left(\mathrm{f}^{*}\right)$ and $\left(\mathrm{f}^{*}\right)^{*}=\mathrm{f}$; (3) If the leader coefficient of $\mathrm{f}$ is a unit, then $(\mathrm{fg})^{*}=\mathrm{f}^{*} \mathrm{~g}^{*}$ and $\overline{\mathrm{f}^{*}}=(\overline{\mathrm{f}})^{*}$;

(4) If $\mathrm{f}$ is a monic basic irreducible polynomial, then $\mathrm{f}^{*}$ is basic irreducible;

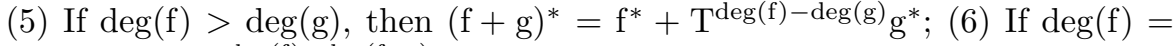
$\operatorname{deg}(\mathrm{g})$, then $\mathrm{T}^{\operatorname{deg}(\mathrm{f})-\operatorname{deg}(\mathrm{f}+\mathrm{g})}(\mathrm{f}+\mathrm{g})^{*}=\mathrm{f}^{*}+\mathrm{g}^{*}$; (7) If the leader coefficients of $\mathrm{f}$ and $\mathrm{g}$ are units and $\mathrm{f}$ and $\mathrm{g}$ are coprime, then $\mathrm{f}^{*}$ and $\mathrm{g}^{*}$ are coprime; (8) If $\delta_{1}, \ldots, \delta_{k} \in \mathrm{R}[\mathrm{T}]$, then $\left(\delta_{1}+\ldots+\delta_{k}\right)^{*}=\mathrm{T}^{u_{1}} \delta_{1}^{*}+\ldots+\mathrm{T}^{u_{k}} \delta_{k}^{*}$, for some $u_{1}, \ldots, u_{k}$ non negative integers; (9) Each polynomial $f_{i}^{*}$ is basic irreducible; (10) Over fields, self reciprocal polynomials have even degree with the only exception of $\mathrm{T}+1$; (11) For $1 \leq i \leq r$, the constant coefficient of $\mathrm{f}_{i}$ is a unit, $\operatorname{deg}\left(\mathrm{f}_{i}\right)=\operatorname{deg}\left(\mathrm{f}_{i}^{*}\right),\left(\mathrm{f}_{i}^{*}\right)^{*}=\mathrm{f}_{i}$ and $\left(\widehat{\mathrm{f}}_{i}^{*}\right)^{*}=\widehat{\mathrm{f}}_{i} ;(12)\left(\mathrm{f}_{1}^{\circ}\right)^{*}, \ldots,\left(\mathrm{f}_{r}^{\circ}\right)^{*}$ are the unique monic basic irreducible pairwise coprime polynomials such that $\mathrm{T}^{n}-\gamma^{-1}=\left(\mathrm{f}_{1}^{\circ}\right)^{*} \cdots\left(\mathrm{f}_{r}^{\circ}\right)^{*} ;(13)$ If $\mathrm{U}$ is a subset of $\{1, \ldots, r\}$ and $\mathrm{F}=\prod_{u \in \mathrm{U}} \mathrm{f}_{i}$, then $\mathrm{T}^{n}-\gamma=\mathrm{FF}, \mathrm{T}^{n}-\gamma^{-1}=-\gamma^{-1} \mathrm{~F}^{*} \widehat{\mathrm{F}}^{*}$ and $\widehat{\mathrm{F}}^{*}=-\gamma^{-1}{\widehat{\prod_{u \in U}}}_{i}^{*}$, in particular $\widehat{\mathrm{f}_{i}^{*}}=-\gamma^{-1} \widehat{\mathrm{f}}_{i}^{*}, 1 \leq i \leq r$, note that $\widehat{ }{ }^{\wedge}$ in the left side is respect to $\mathrm{T}^{n}-\gamma^{-1}$ and ${ }^{i}$ in the right side is respect to $\mathrm{T}^{n}-\gamma$; (14) If $\gamma=\gamma^{-1}$, then $\mathrm{f}_{i}^{*}$ is an associate of $\mathrm{f}_{j}$ if and only if $\overline{\mathrm{f}_{i}^{*}}$ is an associate of $\overline{\mathrm{f}_{j}}$, in particular $\mathrm{f}_{i}$ is self-reciprocal if and only if $\overline{f_{i}}$ is self-reciprocal; (15) If $\gamma=\gamma^{-1}, \mathrm{f}_{i}$ is a self reciprocal and $\mathrm{f}_{i} \neq \mathrm{T}-1$, then $\mathrm{f}_{i}$ has even degree; (16) If $\gamma=\gamma^{-1}$, after renumbering there are non negative integers $r_{1}, r_{2}$ such that $r=r_{1}+2 r_{2}, \mathrm{f}_{2 i-1}^{*}$ is an associate of $\mathrm{f}_{2 i}, 1 \leq i \leq r_{1}$, and $\mathrm{f}_{2 r_{1}+i}$ is a self-reciprocal polynomial, $1 \leq i \leq r_{2}$.

For the remainder of the manuscript the following notation will be used: (1) When $\gamma=\gamma^{-1}$, the order of the sequence $\mathrm{f}_{1}, \ldots, \mathrm{f}_{r}$ is fixed according to the last assertion; (2) For $u \in\{1, \ldots, r\}, u^{*}$ denotes the index of the polynomial which is associate of $\mathrm{f}_{u}$, observe that $\left(u^{*}\right)^{*}=u$; (3) For $\mathrm{U} \subseteq\{1, \ldots, r\}$, let $\mathrm{U}^{*}=\left\{u^{*}: u \in \mathrm{U}\right\} . \quad \mathrm{U}$ is called self-reciprocal if $\mathrm{U}^{*}=\mathrm{U}$ and special if $\mathrm{U} \cap \mathrm{U}^{*}=\emptyset$. Observe that $\mathrm{U}$ is a self-reciprocal set if and only if $\{1, \ldots, r\} \backslash \mathrm{U}$ is a self-reciprocal set; if $U$ is a special set then $U^{*}$ is a special set and $U \subseteq$ $\left\{1, \ldots, 2 r_{1}\right\} ; \emptyset$ is considered a self-reciprocal and special set. 
SELF DUAL, REVERSIBLE AND COMPLEMENTARY DUALS CONSTACYCLIC CODES OVER FINITE LOCAL FROBENIUS NON-CHAIN RINGS OF LENGTH 5 AND NILPOTENCY INDEX 4

The reciprocal matrix is helpful to determine reversible constacyclic codes and the dual of a constacyclic code, over finite local rings, see [4]. Let $\mathrm{f}, \mathrm{g}, \mathrm{h} \in \mathrm{GF}(q)[\mathrm{T}]$, whit $\mathrm{f}$ irreducible and $\mathrm{gT} \equiv 1 \bmod \langle\mathrm{f}\rangle$, observe that $\mathrm{h}(\mathrm{g}) \equiv \mathrm{g}^{\mathrm{deg}(\mathrm{h})} \mathrm{h}^{*} \bmod \langle\mathrm{f}\rangle$ and $\psi: \mathrm{GF}(q)[\mathrm{T}] /\left\langle\mathrm{f}^{*}\right\rangle \rightarrow \mathrm{GF}(q)[\mathrm{T}] /\langle\mathrm{f}\rangle$ given by $\psi\left(\mathrm{h}+\left\langle\mathrm{f}^{*}\right\rangle\right)=\mathrm{h}(\mathrm{g})+\langle\mathrm{f}\rangle=\mathrm{g}^{\operatorname{deg}(\mathrm{h})} \mathrm{h}^{*}+\langle\mathrm{f}\rangle=\frac{\mathrm{h}^{*}+\langle\mathrm{f}\rangle}{\mathrm{T}^{\operatorname{deg}(\mathrm{h})}+\langle\mathrm{f}\rangle}$ is an isomorphism over $\mathrm{GF}(q)$.

Definition 1. Let $(\mathrm{R}, \mathfrak{m}, \mathrm{GF}(q))$ be a finite local ring, $\mathrm{f} \in \mathrm{R}[\mathrm{T}]$ a monic basic irreducible polynomial, $n \geq \operatorname{deg}(\mathrm{f}), \mathrm{H}=\left(a_{i j}\right)$ a matrix over $\mathrm{GF}\left(q^{\operatorname{deg}(\mathrm{f})}\right)=$ $\mathrm{GF}(q)[\mathrm{T}] /\left\langle\overline{\mathrm{f}^{*}}\right\rangle$ and $\psi: \mathrm{GF}(q)[\mathrm{T}] /\left\langle\overline{\mathrm{f}^{*}}\right\rangle \rightarrow \mathrm{GF}(q)[\mathrm{T}] /\langle\overline{\mathrm{f}}\rangle$ as above. The matrix $\left(\overline{\mathrm{T}}^{n} \psi\left(a_{i j}\right)\right)$ over $\mathrm{GF}\left(q^{\operatorname{deg}(\mathrm{f})}\right)=\mathrm{GF}(q)[\mathrm{T}] /\langle\overline{\mathrm{f}}\rangle$ is called the $n$-th reciprocal matrix of $\mathrm{H}$ with respect $\mathrm{f}$ and is denoted by $\mathrm{H}_{(n, \mathrm{f})}^{*}$.

Under the notation as in Definition 1, from [4], we have the following properties: (1) $(u \mathrm{H})_{(n, \mathrm{f})}^{*}=u \mathrm{H}_{(n, \mathrm{f})}^{*}, u \in \mathrm{GF}(q) ;(2) \mathrm{H}_{(n+r, \mathrm{f})}^{*}=\overline{\mathrm{T}}^{r} \mathrm{H}_{(n, \mathrm{f})}^{*}$; (3) $\left(\mathrm{H}_{(n, \mathrm{f})}^{*}\right)_{\left(n, \mathrm{f}^{*}\right)}^{*}=\mathrm{H}$. Suppose $\gamma$ is a unit of $\mathrm{R}$ and $\mathrm{f}$ is a divisor of $\mathrm{T}^{n}-\gamma$, then: (4) if $\left.\operatorname{deg}(\mathrm{f})=1, \overline{\gamma^{-1}} \mathrm{H}_{(n, \mathrm{f})}^{*}=\mathrm{H} ;(5) \overline{\gamma^{-1}} \mathrm{H}_{(n, \mathrm{f})}^{*}={\overline{\gamma^{-1}}}^{n} \overline{\mathrm{T}}^{n} \psi\left(a_{i j}\right)\right)=\left(\psi\left(a_{i j}\right)\right)$; (6) if $\mathrm{H}$ is in (rre)-form, the matrix $\overline{\gamma^{-1}} \mathrm{H}_{(n, \mathrm{f})}^{*}$ is in (rre)-form.

Observation 2. Let $(\mathrm{R}, \mathrm{m}, \mathrm{GF}(q)), \mathrm{f}, n, \mathrm{H}=\left(a_{i j}\right), \psi$ as in Definition 1 and $\gamma$ a unit of $\mathrm{R}$. Suppose $\mathrm{f}$ is self reciprocal, $\operatorname{deg}(\mathrm{f})=2$ s and $\mathrm{f} \mid \mathrm{T}^{n}-\gamma$.

(1) $\psi$ is an automorphism of $\mathrm{GF}\left(q^{2 s}\right)$ and $\psi^{2}(\mathrm{~h}+\langle\mathrm{f}\rangle)=\psi\left[\frac{\mathrm{h}^{*}+\langle\mathrm{f}\rangle}{\mathrm{T}^{\operatorname{deg}(\mathrm{h})}+\langle\mathrm{f}\rangle}\right]=$

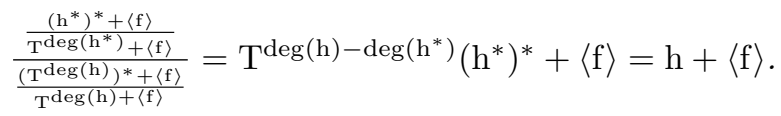

(2) Let $\alpha \in \mathrm{GF}\left(q^{2 s}\right)=\mathrm{GF}(q)[\mathrm{T}] /\langle\mathrm{f}\rangle$ be a root of an irreducible polynomial over $\mathrm{GF}(q)[\mathrm{T}]$ of degree s. Since $\mathrm{GF}\left(q^{2 s}\right)$ has only one automorphism of degree 2 over $\operatorname{GF}(q)$, then this automorphism is $\psi$ and the fixed field of this automorphism is $\operatorname{GF}\left(q^{s}\right)=\{\mathrm{h}(\alpha): \mathrm{h}(\mathrm{T}) \in \mathrm{GF}(q)[\mathrm{T}], \operatorname{deg}(\mathrm{h})<s\}$

(3) $\overline{\gamma^{-1}} \mathrm{H}_{(n, \mathrm{f})}^{*}=\mathrm{H} \Leftrightarrow \mathrm{H}$ is a matrix over $\mathrm{GF}\left(q^{s}\right)$.

The following result will be useful later; claims are treated in Corollary 1 and Lemma 2 of [4].

Lemma 2. Let $(\mathrm{R}, \mathfrak{m}, \mathrm{GF}(q))$ be a finite local ring, $\mathrm{f} \in \mathrm{R}[\mathrm{T}]$ a monic basic irreducible, $\mathrm{K}$ an ideal of $\mathrm{R}$ and $\tilde{\theta}=\left\{\theta_{1}, \ldots, \theta_{l}\right\}$ a minimal $\mathrm{R}$-generating set for K. Let $\vec{\rho}=\left(\rho_{1}, \ldots, \rho_{l}\right), \vec{\nu}=\left(\nu_{1}, \ldots, \nu_{l}\right) \in(\mathrm{R}[\mathrm{T}])^{l}$, where $\vec{\rho}$ is in (rre)form, the leader coefficient of each $\rho_{i}$ is a unit, $n \geq \max \left\{\operatorname{deg}\left(\rho_{i}\right): 1 \leq i \leq l\right\}$ and $\left(\nu_{1}+\langle\mathrm{f}\rangle, \ldots, \nu_{l}+\langle\mathrm{f}\rangle\right)=\left(\rho_{1}+\left\langle\mathrm{f}^{*}\right\rangle, \ldots, \rho_{l}+\left\langle\mathrm{f}^{*}\right\rangle\right)_{(n, \mathrm{f})}^{*}$. There are $u_{1}, u_{2}$ non negative integers such that: 
SELF DUAL, REVERSIBLE AND COMPLEMENTARY DUALS

CONSTACYCLIC CODES OVER FINITE LOCAL FROBENIUS NON-CHAIN

RINGS OF LENGTH 5 AND NILPOTENCY INDEX 4

(1) $\left(\left(\rho_{1} \theta_{1}+\rho_{2} \theta_{2}+\ldots+\rho_{l} \theta_{l}\right)^{*}\right)^{*}=\rho_{1} \theta_{1}+\rho_{2} \theta_{2}+\ldots+\rho_{l} \theta_{l}$;

(2) $\mathrm{T}^{u_{1}}\left(\rho_{1} \theta_{1}+\ldots+\rho_{l} \theta_{l}\right)^{*} \equiv \nu_{1} \theta_{1}+\ldots+\nu_{l} \theta_{l} \bmod \langle\mathfrak{m K}, \mathrm{f}\rangle$;

(3) $\rho_{1} \theta_{1}+\ldots+\rho_{l} \theta_{l} \equiv \mathrm{T}^{u_{2}}\left(\nu_{1} \theta_{1}+\ldots+\nu_{l} \theta_{l}\right)^{*} \bmod \left\langle\mathfrak{m K}, \mathrm{f}^{*}\right\rangle$.

Lemma 3. Let $(\mathrm{R}, \mathfrak{m}, \operatorname{GF}(q)), \mathrm{f}, \operatorname{deg}(\mathfrak{f})=s, \mathbb{T}, \mathbb{T}_{s}, \chi, \tilde{\alpha}=\left\{\alpha_{1}, \ldots, \alpha_{\chi}\right\}$, $\tilde{\beta}=\left\{\beta_{1}, \ldots, \beta_{\chi}\right\}, \mathfrak{F}, \mathrm{J} \in \mathfrak{F}, \ell_{\mathrm{B}}(\mathrm{J})=k+1$, where $k \in\{1, \ldots, \chi-1\}, \mathrm{H}_{\mathrm{J}}=$ $\left(a_{i j}\right), \mathrm{H}_{\mathrm{J}}^{\perp}=\left(b_{i j}\right),\left(\mathrm{H}_{\mathrm{J}}\right)_{\left(n, \mathrm{f}^{*}\right)}^{*}=\left(\nu_{i j}\right),\left(\mathrm{H}_{\mathrm{J}}^{\perp}\right)_{\left(n, \mathrm{f}^{*}\right)}^{*}=\left(\mu_{i j}\right)$, as in Observation 1. Let $n$ an integer with $n \geq \operatorname{deg}(\mathrm{f}),\left(\mathrm{B}=\mathrm{R}[\mathrm{T}] /\langle\mathrm{f}\rangle, \mathfrak{m B}, \mathrm{GF}\left(q^{s}\right)\right),\left(\mathrm{B}^{*}=\right.$ $\left.\mathrm{R}[\mathrm{T}] /\left\langle\mathrm{f}^{*}\right\rangle, \mathrm{mB}^{*}, \mathrm{GF}\left(q^{s}\right)\right)$ the separable extensions of $\mathrm{R}$ determined by $\mathrm{f}$ and $\mathrm{f}^{*}$, respectively. We have the following:

(1) Let $\pi \in\{1, \ldots, k\}$ and $\varpi \in\{1, \ldots, \chi-k\}$, the following relations hold in the ring $\mathrm{B}$ :

$$
\begin{aligned}
& \left(\nu_{\pi 1}^{\mathbb{T}_{s}} \beta_{1}+\ldots+\nu_{\pi \chi}^{\mathbb{T}_{s}} \beta_{\chi}\right)^{*}\left(b_{\varpi 1}^{\mathbb{T}_{s}} \alpha_{1}+\ldots+b_{\varpi}^{\mathbb{T}_{s}} \alpha_{\chi}\right)=0, \\
& \left(a_{\pi 1}^{\mathbb{T}_{s}} \beta_{1}+\ldots+a_{\pi \chi}^{\mathbb{T}_{s}} \beta_{\chi}\right)\left(\mu_{\varpi 1}^{\mathbb{T}_{s}} \alpha_{1}+\ldots+\mu_{\varpi \chi}^{\mathbb{T}_{s}} \alpha_{\chi}\right)^{*}=0 .
\end{aligned}
$$

(2) The following relation holds in the ring $\mathrm{B}^{*}$ :

$$
\begin{gathered}
\left\langle\left(a_{11}^{\mathbb{T}_{s}} \beta_{1}+\ldots+a_{1 \chi}^{\mathbb{T}_{s}} \beta_{\chi}\right)^{*}, \ldots,\left(a_{k 1}^{\mathbb{T}_{s}} \beta_{1}+\ldots+a_{k \chi}^{\mathbb{T}_{s}} \beta_{\chi}\right)^{*}\right\rangle+\operatorname{ann}_{\mathrm{R}}(\mathfrak{m}) \mathrm{B}^{*}= \\
\left\langle\nu_{11}^{\mathbb{T}_{s}} \beta_{1}+\ldots+\nu_{1 \chi}^{\mathbb{T}_{s}} \beta_{\chi}, \ldots, \nu_{k 1}^{\mathbb{T}_{s}} \beta_{1}+\ldots+\nu_{k \chi}^{\mathbb{T}_{s}} \beta_{\chi}\right\rangle+\operatorname{ann}_{\mathrm{R}}(\mathfrak{m}) \mathrm{B}^{*} .
\end{gathered}
$$

(3) Let $\phi \in \mathrm{R}[\mathrm{T}]$ such that $\phi+\langle\mathrm{f}\rangle \in \mathrm{J}=\left(\mathrm{H}_{\mathrm{J}}\right)_{\tilde{\beta}}^{\mathbb{T}_{s}}$, then there exist $\mathrm{h}_{1}, \ldots, \mathrm{h}_{k} \in$ $\mathrm{R}[\mathrm{T}]$ such that:

$\phi^{*} \equiv \mathrm{h}_{1}\left(\nu_{11}^{\mathbb{T}_{s}} \beta_{1}+\ldots+\nu_{1 \chi}^{\mathbb{T}_{s}} \beta_{\chi}\right)+\ldots+\mathrm{h}_{k}\left(\nu_{k 1}^{\mathbb{T}_{s}} \beta_{1}+\ldots+\nu_{k \chi}^{\mathbb{T}_{s}} \beta_{\chi}\right) \bmod \left\langle\operatorname{ann}_{\mathrm{R}}(\mathfrak{m}), \mathrm{f}^{*}\right\rangle$.

In particular $\phi^{*}+\left\langle\mathrm{f}^{*}\right\rangle \in\left(\left(\mathrm{H}_{\mathrm{J}}\right)_{\left(n, \mathrm{f}^{*}\right)}^{*}\right)_{\tilde{\beta}}^{\mathbb{T}_{s}}$, in the ring $\mathrm{B}^{*}$.

Proof: Observe that $\mathrm{T}$ is a unit of the rings $\mathrm{B}$ and $\mathrm{B}^{*}$.

(1) By (3) of Lemma 2, there are non negative integer $u_{1}, u_{2}$ such that the following relations hold in $\mathrm{B}$ :

(a) $a_{\pi 1}^{\mathbb{T}_{s}} \beta_{1}+\ldots+a_{\pi \chi}^{\mathbb{T}_{s}} \beta_{\chi} \equiv \mathrm{T}^{u_{1}}\left(\nu_{\pi 1}^{\mathbb{T}_{s}} \beta_{1}+\ldots+\nu_{\pi \chi}^{\mathbb{T}_{s}} \beta_{\chi}\right)^{*} \bmod \operatorname{mann}_{\mathrm{R}}\left(\mathfrak{m}^{2}\right) \mathrm{B}=$ $\operatorname{ann}_{\mathrm{R}}(\mathfrak{m}) \mathrm{B}$

(b) $b_{\varpi 1}^{\mathbb{T}_{s}} \alpha_{1}+\ldots+b_{\varpi \chi}^{\mathbb{T}_{s}} \alpha_{\chi} \equiv \mathrm{T}^{u_{2}}\left(\mu_{\varpi 1}^{\mathbb{T}_{s}} \alpha_{1}+\ldots+\mu_{\varpi \chi}^{\mathbb{T}_{s}} \alpha_{\chi}\right)^{*} \bmod \mathfrak{m}^{2} \mathrm{~B}$,

From (4c) of Observation 1 and (a) and (b) we have

$$
\begin{aligned}
& 0=\left(a_{\pi 1}^{\mathbb{T}_{s}} \beta_{1}+\ldots+a_{\pi \chi}^{\mathbb{T}_{s}} \beta_{\chi}\right)\left(b_{\varpi 1}^{\mathbb{T}_{s}} \alpha_{1}+\ldots+b_{\varpi \chi}^{\mathbb{T}_{s}} \alpha_{\chi}\right)= \\
& \mathrm{T}^{u_{1}}\left(\nu_{\pi 1}^{\mathbb{T}_{s}} \beta_{1}+\ldots+\nu_{\pi \chi}^{\mathbb{T}_{s}} \beta_{\chi}\right)^{*}\left(b_{\varpi 1}^{\mathbb{T}_{s}} \alpha_{1}+\ldots+b_{\varpi \chi}^{\mathbb{T}_{s}} \alpha_{\chi}\right)= \\
& \mathrm{T}^{u_{2}}\left(a_{\pi 1}^{\mathbb{T}_{s}} \beta_{1}+\ldots+a_{\pi \chi}^{\mathbb{T}_{s}} \beta_{\chi}\right)\left(\mu_{\varpi 1}^{\mathbb{T}_{s}} \alpha_{1}+\ldots+\mu_{\varpi \chi}^{\mathbb{T}_{s}} \alpha_{\chi}\right)^{*} .
\end{aligned}
$$


SELF DUAL, REVERSIBLE AND COMPLEMENTARY DUALS

CONSTACYCLIC CODES OVER FINITE LOCAL FROBENIUS NON-CHAIN

RINGS OF LENGTH 5 AND NILPOTENCY INDEX 4

(2) From (2) of Lemma 2, there are units of the ring $\mathrm{B}^{*}, \sigma_{1}, \ldots, \sigma_{k}$, such that

$$
\begin{gathered}
\left(a_{11}^{\mathbb{T}_{s}} \beta_{1}+\ldots+a_{1 \chi}^{\mathbb{T}_{s}} \beta_{\chi}\right)^{*} \equiv \sigma_{1}\left(\nu_{11}^{\mathbb{T}_{s}} \beta_{1}+\ldots+\nu_{1 \chi}^{\mathbb{T}_{s}} \beta_{\chi}\right) \bmod \left\langle\operatorname{ann}_{\mathrm{R}}(\mathfrak{m}), \mathrm{f}^{*}\right\rangle, \\
\vdots \\
\left(a_{k 1}^{\mathbb{T}_{s}} \beta_{1}+\ldots+a_{k \chi}^{\mathbb{T}_{s}} \beta_{\chi}\right)^{*} \equiv \sigma_{k}\left(\nu_{k 1}^{\mathbb{T}_{s}} \beta_{1}+\ldots+\nu_{k \chi}^{\mathbb{T}_{s}} \beta_{\chi}\right) \bmod \left\langle\operatorname{ann}_{\mathrm{R}}(\mathfrak{m}), \mathrm{f}^{*}\right\rangle,
\end{gathered}
$$

the assertion follows.

(3) There are $\phi_{1}, \ldots, \phi_{k} \in \mathrm{R}[\mathrm{T}]$ such that

$\phi \equiv \phi_{1}\left(a_{11}^{\mathbb{T}_{s}} \beta_{1}+\ldots+a_{1 \chi}^{\mathbb{T}_{s}} \beta_{\chi}\right)+\ldots+\phi_{k}\left(a_{k 1}^{\mathbb{T}_{s}} \beta_{1}+\ldots+a_{k \chi}^{\mathbb{T}_{s}} \beta_{\chi}\right) \bmod \left\langle\operatorname{ann}_{\mathrm{R}}(\mathfrak{m}), \mathrm{f}\right\rangle$,

then there are $v_{1}, \ldots, v_{k}$ non negative integers such that

$$
\begin{aligned}
& \phi_{1}^{*} \mathrm{~T}^{v_{1}}\left(a_{11}^{\mathbb{T}_{s}} \beta_{1}+\ldots+a_{1 \chi}^{\mathbb{T}_{s}} \beta_{\chi}\right)^{*}+\ldots+\phi_{k}^{*} \mathrm{~T}^{v_{k}}\left(a_{k 1}^{\mathbb{T}_{s}} \beta_{1}+\ldots+a_{k \chi}^{\mathbb{T}_{s}} \beta_{\chi}\right)^{*} \\
\equiv & \phi_{1}^{*} \sigma_{1} \mathrm{~T}^{v_{1}}\left(\nu_{11}^{\mathbb{T}_{s}} \beta_{1}+\ldots+\nu_{1 \chi}^{\mathbb{T}_{s}} \beta_{\chi}\right)+\ldots+\phi_{k}^{*} \sigma_{k} \mathrm{~T}^{v_{k}}\left(\nu_{k 1}^{\mathbb{T}_{s}} \beta_{1}+\ldots+\nu_{k \chi}^{\mathbb{T}_{s}} \beta_{\chi}\right) \\
\equiv & \phi^{*} \bmod \left\langle\operatorname{ann}_{\mathrm{R}}(\mathfrak{m}), \mathrm{f}^{*}\right\rangle .
\end{aligned}
$$

Lemma 4. Let $(\mathrm{R}, \mathfrak{m}, \mathrm{GF}(q))$, f, B, B* $\mathbb{T}, \mathbb{T}_{s}, \chi, \tilde{\alpha}=\left\{\alpha_{1}, \ldots, \alpha_{\chi}\right\}, \tilde{\beta}=$ $\left\{\beta_{1}, \ldots, \beta_{\chi}\right\}$ and $n$ as in Lemma 3. Let $\mathfrak{G}$ as in Observation $1, \mathrm{I} \in \mathfrak{G}$ with $\ell_{\mathrm{B}}(\mathrm{I})=k+\ell_{\mathrm{R}}\left(\mathfrak{m}^{2}\right), k \in\{1, \ldots, \chi-1\}, \mathrm{H}_{\mathrm{I}}=\left(c_{i j}\right), \mathrm{H}_{\mathrm{I}}^{\perp}=\left(d_{i j}\right),\left(\mathrm{H}_{\mathrm{I}}\right)_{\left(n, \mathrm{f}^{*}\right)}^{*}=$ $\left(\varsigma_{i j}\right)$ and $\left(\mathrm{H}_{\mathrm{I}}^{\perp}\right)_{\left(n, \mathrm{f}^{*}\right)}^{*}=\left(\epsilon_{i j}\right)$. We have the following:

(1) Let $\pi \in\{1, \ldots, k\}$ and $\varpi \in\{1, \ldots, \chi-k\}$, the following relations hold in the ring $\mathrm{B}$ :

$$
\begin{aligned}
& \left(\varsigma_{\pi 1}^{\mathbb{T}_{s}} \alpha_{1}+\ldots+\varsigma_{\pi \chi}^{\mathbb{T}_{s}} \alpha_{\chi}\right)^{*}\left(d_{\varpi 1}^{\mathbb{T}_{s}} \beta_{1}+\ldots+d_{\varpi \chi}^{\mathbb{T}_{s}} \beta_{\chi}\right)=0, \\
& \left(c_{\pi 1}^{\mathbb{T}_{s}} \alpha_{1}+\ldots+c_{\pi \chi}^{\mathbb{T}_{s}} \alpha_{\chi}\right)\left(\epsilon_{\varpi 1}^{\mathbb{T}_{s}} \beta_{1}+\ldots+\epsilon_{\varpi \chi}^{\mathbb{T}_{s}} \beta_{\chi}\right)^{*}=0 .
\end{aligned}
$$

(2) The following relation holds in the ring $\mathrm{B}^{*}$ :

$$
\begin{gathered}
\left\langle\left(c_{11}^{\mathbb{T}_{s}} \alpha_{1}+\ldots+c_{1 \chi}^{\mathbb{T}_{s}} \alpha_{\chi}\right)^{*}, \ldots,\left(c_{k 1}^{\mathbb{T}_{s}} \alpha_{1}+\ldots+c_{k \chi}^{\mathbb{T}_{s}} \alpha_{\chi}\right)^{*}\right\rangle+\mathfrak{m}^{2} \mathrm{~B}^{*}= \\
\left\langle\varsigma_{11}^{\mathbb{T}_{s}} \alpha_{1}+\ldots+\varsigma_{1 \chi}^{\mathbb{T}_{s}} \alpha_{\chi}, \ldots, \varsigma_{k 1}^{\mathbb{T}_{s}} \alpha_{1}+\ldots+\varsigma_{k \chi}^{\mathbb{T}_{s}} \alpha_{\chi}\right\rangle+\mathfrak{m}^{2} \mathrm{~B}^{*}
\end{gathered}
$$

(3) Let $\phi \in \mathrm{R}[\mathrm{T}]$ such that $\phi+\langle\mathrm{f}\rangle \in \mathrm{I}=\left(\mathrm{H}_{\mathrm{I}}\right)_{\tilde{\alpha}}^{\mathbb{T} s}+\mathfrak{m}^{2} \mathrm{~B}$, then there exist $\mathrm{h}_{1}, \ldots, \mathrm{h}_{k} \in \mathrm{R}[\mathrm{T}]$ such that

$$
\phi^{*} \equiv \mathrm{h}_{1}\left(\varsigma_{11}^{\mathbb{T}_{s}} \alpha_{1}+\ldots+\varsigma_{1 \chi}^{\mathbb{T}_{s}} \alpha_{\chi}\right)+\ldots+\mathrm{h}_{k}\left(\varsigma_{k 1}^{\mathbb{T}_{s}} \alpha_{1}+\ldots+\varsigma_{k \chi}^{\mathbb{T}_{s}} \alpha_{\chi}\right) \bmod \left\langle\mathfrak{m}^{2}, \mathrm{f}^{*}\right\rangle .
$$

In particular $\phi^{*}+\left\langle\mathrm{f}^{*}\right\rangle \in\left(\left(\mathrm{H}_{\mathrm{I}}\right)_{\left(n, \mathrm{f}^{*}\right)}^{*}\right)_{\tilde{\alpha}}^{\mathbb{T}} s+\mathfrak{m}^{2} \mathrm{~B}^{*}$, in the ring $\mathrm{B}^{*}$. 
SELF DUAL, REVERSIBLE AND COMPLEMENTARY DUALS

CONSTACYCLIC CODES OVER FINITE LOCAL FROBENIUS NON-CHAIN

Proof: Observe that $\mathrm{T}$ is a unit of the rings $\mathrm{B}$ and $\mathrm{B}^{*}$.

(1) By (3) of Lemma 2, there are non negative integer $u_{1}, u_{2}$ such that

(a) $c_{\pi 1}^{\mathbb{T}_{s}} \alpha_{1}+\ldots+c_{\pi \chi}^{\mathbb{T}_{s}} \alpha_{\chi} \equiv \mathrm{T}^{u_{1}}\left(\varsigma_{\pi 1}^{\mathbb{T}_{s}} \alpha_{1}+\ldots+\varsigma_{\pi \chi}^{\mathbb{T}_{s}} \alpha_{\chi}\right)^{*} \bmod \mathfrak{m}^{2} \mathrm{~B}$,

(b) $d_{\varpi 1}^{\mathbb{T}_{s}} \beta_{1}+\ldots+d_{\varpi \chi}^{\mathbb{T}_{s}} \beta_{\chi} \equiv \mathrm{T}^{u_{2}}\left(\epsilon_{\varpi 1}^{\mathbb{T}_{s}} \beta_{1}+\ldots+\epsilon_{\varpi \chi}^{\mathbb{T}_{s}} \beta_{\chi}\right)^{*} \bmod \operatorname{mann}_{\mathrm{R}}\left(\mathfrak{m}^{2}\right) \mathrm{B}=$ $\operatorname{ann}_{\mathrm{R}}(\mathfrak{m}) \mathrm{B}$.

From (7) of Observation 1 and (a) and (b) we have

$$
\begin{aligned}
& 0=\left(c_{\pi 1}^{\mathbb{T}_{s}} \alpha_{1}+\ldots+c_{\pi \chi}^{\mathbb{T}_{s}} \alpha_{\chi}\right)\left(d_{\varpi 1}^{\mathbb{T}_{s}} \beta_{1}+\ldots+d_{\varpi \chi}^{\mathbb{T}_{s}} \beta_{\chi}\right)= \\
& \mathrm{T}^{u_{1}}\left(\varsigma_{\pi 1}^{\mathbb{T}_{s}} \alpha_{1}+\ldots+\varsigma_{\pi \chi}^{\mathbb{T}_{s}} \alpha_{\chi}\right)^{*}\left(d_{\varpi 1}^{\mathbb{T}_{s}} \beta_{1}+\ldots+d_{\varpi \chi}^{\mathbb{T}_{s}} \beta_{\chi}\right)= \\
& \mathrm{T}^{u_{2}}\left(c_{\pi 1}^{\mathbb{T}_{s}} \alpha_{1}+\ldots+c_{\pi \chi}^{\mathbb{T}_{s}} \alpha_{\chi}\right)\left(\epsilon_{\varpi 1}^{\mathbb{T}_{s}} \beta_{1}+\ldots+\epsilon_{\varpi \chi}^{\mathbb{T}_{s}} \beta_{\chi}\right)^{*},
\end{aligned}
$$

the assertion follows.

(2) From (2) of Lemma 2, there are units of the ring $\mathrm{B}^{*}, \sigma_{1}, \ldots, \sigma_{k}$, such that

$$
\begin{gathered}
\left(c_{11}^{\mathbb{T}_{s}} \alpha_{1}+\ldots+c_{1 \chi}^{\mathbb{T}_{s}} \alpha_{\chi}\right)^{*} \equiv \sigma_{1}\left(\varsigma_{11}^{\mathbb{T}_{s}} \alpha_{1}+\ldots+\varsigma_{1 \chi}^{\mathbb{T}_{s}} \alpha_{\chi}\right) \bmod \left\langle\mathfrak{m}^{2}, \mathrm{f}^{*}\right\rangle \\
\vdots \\
\left(c_{k 1}^{\mathbb{T}_{s}} \alpha_{1}+\ldots+c_{k \chi}^{\mathbb{T}_{s}} \alpha_{\chi}\right)^{*} \equiv \sigma_{k}\left(\varsigma_{k 1}^{\mathbb{T}_{s}} \alpha_{1}+\ldots+\varsigma_{k \chi}^{\mathbb{T}_{s}} \alpha_{\chi}\right) \bmod \left\langle\mathfrak{m}^{2}, \mathrm{f}^{*}\right\rangle
\end{gathered}
$$

the assertion follows.

(3) There are $\phi_{1}, \ldots, \phi_{k} \in \mathrm{R}[\mathrm{T}]$ such that

$$
\phi \equiv \phi_{1}\left(c_{11}^{\mathbb{T}_{s}} \alpha_{1}+\ldots+c_{1 \chi}^{\mathbb{T}_{s}} \alpha_{\chi}\right)+\ldots+\phi_{k}\left(c_{k 1}^{\mathbb{T}_{s}} \alpha_{1}+\ldots+c_{k \chi}^{\mathbb{T}_{s}} \alpha_{\chi}\right) \bmod \left\langle\mathfrak{m}^{2}, \mathrm{f}\right\rangle,
$$

then there are $v_{1}, \ldots, v_{k}$ non negative integers such that

$$
\begin{aligned}
& \phi_{1}^{*} \mathrm{~T}^{v_{1}}\left(c_{11}^{\mathbb{T}_{s}} \alpha_{1}+\ldots+c_{1 \chi}^{\mathbb{T}_{s}} \alpha_{\chi}\right)^{*}+\ldots+\phi_{k}^{*} \mathrm{~T}^{v_{k}}\left(c_{k 1}^{\mathbb{T}_{s}} \alpha_{1}+\ldots+c_{k \chi}^{\mathbb{T}_{s}} \alpha_{\chi}\right)^{*} \\
\equiv & \phi_{1}^{*} \sigma_{1} \mathrm{~T}^{v_{1}}\left(\varsigma_{11}^{\mathbb{T}_{s}} \alpha_{1}+\ldots+\varsigma_{1 \chi}^{\mathbb{T}_{s}} \alpha_{\chi}\right)+\ldots+\phi_{k}^{*} \sigma_{k} \mathrm{~T}^{v_{k}}\left(\varsigma_{k 1}^{\mathbb{T}_{s}} \alpha_{1}+\ldots+\varsigma_{k \chi}^{\mathbb{T}_{s}} \alpha_{\chi}\right) \\
\equiv & \phi^{*} \bmod \left\langle\mathfrak{m}^{2}, \mathrm{f}^{*}\right\rangle .
\end{aligned}
$$

\subsection{The family $\mathfrak{F}_{5}^{4}$}

The family of finite local Frobenius non-chain rings of length 5 whose maximal ideal has nilpotency index 4 is denoted by $\mathfrak{F}_{5}^{4}$. Recently, the rings in the family $\mathfrak{F}_{5}^{4}$ and the lattice of ideals of a separable extension of a ring in this family were determined in [5] and for completeness we recall the results here.

Theorem 1. Let $\left(\mathrm{R}, \mathfrak{m}, \mathrm{GF}\left(p^{d}\right)\right) \in \mathfrak{F}_{5}^{4}$, then $\mathrm{R}$ is isomorphic to one of the following rings:

If $p$ is odd 
SELF DUAL, REVERSIBLE AND COMPLEMENTARY DUALS

CONSTACYCLIC CODES OVER FINITE LOCAL FROBENIUS NON-CHAIN

RINGS OF LENGTH 5 AND NILPOTENCY INDEX 4

(i) $\operatorname{char}(\mathrm{R})=p^{2}$ and $\left\{0,1, \zeta, \ldots, \zeta^{2^{d}-2}\right\}$ is the Teichmüller set of the Galois $\operatorname{ring} \operatorname{GR}\left(p^{2}, d\right)$.

(a) $\mathfrak{m}^{2}=\langle p\rangle=\left\langle\mathrm{y}^{2}\right\rangle$ and $\mathfrak{m}^{3}=\left\langle\mathrm{y}^{3}\right\rangle=\left\langle\mathrm{x}^{2}\right\rangle$.

(1) $\operatorname{GR}\left(p^{2}, d\right)[\mathrm{X}, \mathrm{Y}] /\left\langle\mathrm{Y}^{2}-p, \mathrm{X}^{2}-\mathrm{Y}^{3}, \mathrm{XY}\right\rangle$,

(2) $\operatorname{GR}\left(p^{2}, d\right)[\mathrm{X}, \mathrm{Y}] /\left\langle\zeta \mathrm{Y}^{2}-p, \mathrm{X}^{2}-\mathrm{Y}^{3}, \mathrm{XY}\right\rangle$,

(3) $\operatorname{GR}\left(p^{2}, d\right)[\mathrm{X}, \mathrm{Y}] /\left\langle\mathrm{Y}^{2}-p, \mathrm{X}^{2}-\zeta \mathrm{Y}^{3}, \mathrm{XY}\right\rangle$,

(4) $\operatorname{GR}\left(p^{2}, d\right)[\mathrm{X}, \mathrm{Y}] /\left\langle\zeta \mathrm{Y}^{2}-p, \mathrm{X}^{2}-\zeta \mathrm{Y}^{3}, \mathrm{XY}\right\rangle$.

(b) $\mathfrak{m}^{2}=\left\langle\mathrm{y}^{2}\right\rangle, \mathfrak{m}^{3}=\langle p\rangle=\left\langle\mathrm{y}^{3}\right\rangle=\left\langle\mathrm{x}^{2}\right\rangle$ and $3 \mid p^{d}-1$.

(5) $\mathrm{GR}\left(p^{2}, d\right)[\mathrm{X}, \mathrm{Y}] /\left\langle\mathrm{Y}^{3}-p, \mathrm{X}^{2}-\mathrm{Y}^{3}, \mathrm{XY}\right\rangle$,

(6) $\operatorname{GR}\left(p^{2}, d\right)[\mathrm{X}, \mathrm{Y}] /\left\langle\zeta \mathrm{Y}^{3}-p, \mathrm{X}^{2}-\mathrm{Y}^{3}, \mathrm{XY}\right\rangle$,

(7) $\operatorname{GR}\left(p^{2}, d\right)[\mathrm{X}, \mathrm{Y}] /\left\langle\zeta^{2} \mathrm{Y}^{3}-p, \mathrm{X}^{2}-\mathrm{Y}^{3}, \mathrm{XY}\right\rangle$,

(8) $\operatorname{GR}\left(p^{2}, d\right)[\mathrm{X}, \mathrm{Y}] /\left\langle\mathrm{Y}^{3}-p, \mathrm{X}^{2}-\zeta \mathrm{Y}^{3}, \mathrm{XY}\right\rangle$,

(9) $\operatorname{GR}\left(p^{2}, d\right)[\mathrm{X}, \mathrm{Y}] /\left\langle\zeta \mathrm{Y}^{3}-p, \mathrm{X}^{2}-\zeta \mathrm{Y}^{3}, \mathrm{XY}\right\rangle$,

(10) $\operatorname{GR}\left(p^{2}, d\right)[\mathrm{X}, \mathrm{Y}] /\left\langle\zeta^{2} \mathrm{Y}^{3}-p, \mathrm{X}^{2}-\zeta \mathrm{Y}^{3}, \mathrm{XY}\right\rangle$.

(c) $\mathfrak{m}^{2}=\left\langle\mathrm{y}^{2}\right\rangle, \mathfrak{m}^{3}=\langle p\rangle=\left\langle\mathrm{y}^{3}\right\rangle=\left\langle\mathrm{x}^{2}\right\rangle$ and $3 \not p p^{d}-1$.

(11) $\mathrm{GR}\left(p^{2}, d\right)[\mathrm{X}, \mathrm{Y}] /\left\langle\mathrm{Y}^{3}-p, \mathrm{X}^{2}-\mathrm{Y}^{3}, \mathrm{XY}\right\rangle$,

(12) $\mathrm{GR}\left(p^{2}, d\right)[\mathrm{X}, \mathrm{Y}] /\left\langle\mathrm{Y}^{3}-p, \mathrm{X}^{2}-\zeta \mathrm{Y}^{3}, \mathrm{XY}\right\rangle$.

(ii) $\operatorname{char}(\mathrm{R})=p$

(a) $\mathfrak{m}^{2}=\left\langle\mathrm{y}^{2}\right\rangle$ and $\mathfrak{m}^{3}=\left\langle\mathrm{y}^{3}\right\rangle=\left\langle\mathrm{x}^{2}\right\rangle$.

(13) $\mathrm{GF}\left(p^{d}\right)[\mathrm{X}, \mathrm{Y}] /\left\langle\mathrm{X}^{2}-\mathrm{Y}^{3}, \mathrm{XY}\right\rangle$.

(iii) $\operatorname{char}(\mathrm{R})=p^{4}$ and $\left\{0,1, \zeta, \ldots, \zeta^{p^{d}-2}\right\}$ is the Teichmüller set of the Galois ring $\operatorname{GR}\left(p^{4}, d\right)$.

(a) $p \notin \mathfrak{m}^{2}, \mathfrak{m}^{2}=\left\langle p^{2}\right\rangle$ and $\mathfrak{m}^{3}=\left\langle p^{3}\right\rangle=\left\langle\mathrm{x}^{2}\right\rangle$.

(14) $\operatorname{GR}\left(p^{4}, d\right)[\mathrm{X}] /\left\langle\mathrm{X}^{2}-p^{3}, p \mathrm{X}\right\rangle$,

(15) $\mathrm{GR}\left(p^{4}, d\right)[\mathrm{X}] /\left\langle\mathrm{X}^{2}-\zeta p^{3}, p \mathrm{X}\right\rangle$.

(iv) $\operatorname{char}(\mathrm{R})=p^{3}$ and $\left\{0,1, \zeta, \ldots, \zeta^{p^{d}-2}\right\}$ is the Teichmüller set of the Galois ring $\operatorname{GR}\left(p^{3}, d\right)$.

(a) $p \notin \mathfrak{m}^{2}, \mathfrak{m}^{2}=\left\langle\mathrm{x}^{2}\right\rangle, \mathfrak{m}^{3}=\left\langle p^{2}\right\rangle=\left\langle\mathrm{x}^{3}\right\rangle$ and $3 \not p^{d}-1$.

(16) $\operatorname{GR}\left(p^{3}, d\right)[\mathrm{X}] /\left\langle p^{2}-\mathrm{X}^{3}, p \mathrm{X}\right\rangle$.

(b) $p \notin \mathfrak{m}^{2}, \mathfrak{m}^{2}=\left\langle\mathrm{x}^{2}\right\rangle, \mathfrak{m}^{3}=\left\langle p^{2}\right\rangle=\left\langle\mathrm{x}^{3}\right\rangle$ and $3 \mid p^{d}-1$,

(17) $\operatorname{GR}\left(p^{3}, d\right)[\mathrm{X}] /\left\langle p^{2}-\mathrm{X}^{3}, p \mathrm{X}\right\rangle$,

(18) $\operatorname{GR}\left(p^{3}, d\right)[\mathrm{X}] /\left\langle p^{2}-\zeta \mathrm{X}^{3}, p \mathrm{X}\right\rangle$,

(19) $\operatorname{GR}\left(p^{3}, d\right)[\mathrm{X}] /\left\langle p^{2}-\zeta^{2} \mathrm{X}^{3}, p \mathrm{X}\right\rangle$.

If $p=2$ 
(i) $\operatorname{char}(\mathrm{R})=2^{2}$ and $\left\{0,1, \zeta, \ldots, \zeta^{2^{d}-2}\right\}$ is the Teichmüller set of the Galois ring $\operatorname{GR}\left(2^{2}, d\right)$.

(a) $\mathfrak{m}^{2}=\langle 2\rangle=\left\langle\mathrm{y}^{2}\right\rangle$ and $\mathfrak{m}^{3}=\left\langle\mathrm{x}^{2}\right\rangle=\left\langle\mathrm{y}^{3}\right\rangle$.

(20) $\mathrm{GR}\left(2^{2}, d\right)[\mathrm{X}, \mathrm{Y}] /\left\langle\mathrm{Y}^{2}-2, \mathrm{X}^{2}-\mathrm{Y}^{3}, \mathrm{XY}\right\rangle$.

(b) $\mathfrak{m}^{2}=\left\langle\mathrm{y}^{2}\right\rangle, \mathfrak{m}^{3}=\langle 2\rangle=\left\langle\mathrm{x}^{2}\right\rangle=\left\langle\mathrm{y}^{3}\right\rangle$ and $3 \mid 2^{d}-1$.

(21) $\mathrm{GR}\left(2^{2}, d\right)[\mathrm{X}, \mathrm{Y}] /\left\langle\mathrm{Y}^{3}-2, \mathrm{X}^{2}-\mathrm{Y}^{3}, \mathrm{XY}\right\rangle$,

(22) $\mathrm{GR}\left(2^{2}, d\right)[\mathrm{X}, \mathrm{Y}] /\left\langle\zeta \mathrm{Y}^{3}-2, \mathrm{X}^{2}-\mathrm{Y}^{3}, \mathrm{XY}\right\rangle$,

(23) $\mathrm{GR}\left(2^{2}, d\right)[\mathrm{X}, \mathrm{Y}] /\left\langle\zeta^{2} \mathrm{Y}^{3}-2, \mathrm{X}^{2}-\mathrm{Y}^{3}, \mathrm{XY}\right\rangle$.

(c) $\mathfrak{m}^{2}=\left\langle\mathrm{y}^{2}\right\rangle, \mathfrak{m}^{3}=\langle 2\rangle=\left\langle\mathrm{x}^{2}\right\rangle=\left\langle\mathrm{y}^{3}\right\rangle$ and $3 \chi^{d}-1$.

(24) $\mathrm{GR}\left(2^{2}, d\right)[\mathrm{X}, \mathrm{Y}] /\left\langle\mathrm{Y}^{3}-2, \mathrm{X}^{2}-\mathrm{Y}^{3}, \mathrm{XY}\right\rangle$.

(ii) $\operatorname{char}(\mathrm{R})=2$

(a) $\mathfrak{m}^{2}=\left\langle\mathrm{y}^{2}\right\rangle$ and $\mathfrak{m}^{3}=\left\langle\mathrm{x}^{2}\right\rangle=\left\langle\mathrm{y}^{3}\right\rangle$.

(25) $\mathrm{GF}\left(2^{d}\right)[\mathrm{X}, \mathrm{Y}] /\left\langle\mathrm{X}^{2}-\mathrm{Y}^{3}, \mathrm{XY}\right\rangle$

(iii) $\operatorname{char}(\mathrm{R})=2^{4}$.

(a) $\mathfrak{m}^{2}=\left\langle 2^{2}\right\rangle, \mathfrak{m}^{3}=\left\langle 2^{3}\right\rangle=\left\langle\mathrm{x}^{2}\right\rangle$ and $2 \notin \mathfrak{m}^{2}$.

(26) $\mathrm{GR}\left(2^{4}, d\right)[\mathrm{X}] /\left\langle\mathrm{X}^{2}-2^{3}, 2 \mathrm{X}\right\rangle$.

(iv) $\operatorname{char}(\mathrm{R})=2^{3}$ and $\left\{0,1, \zeta, \ldots, \zeta^{2^{d}-2}\right\}$ is the Teichmüller set of the Galois ring $\operatorname{GR}\left(2^{3}, d\right)$.

(a) $2 \notin \mathfrak{m}^{2}, \mathfrak{m}^{2}=\left\langle\mathrm{x}^{2}\right\rangle, \mathfrak{m}^{3}=\left\langle 2^{2}\right\rangle=\left\langle\mathrm{x}^{3}\right\rangle$ and $3 \not 2^{d}-1$.

(27) $\mathrm{GR}\left(2^{3}, d\right)[\mathrm{X}] /\left\langle 2^{2}-\mathrm{X}^{3}, 2 \mathrm{X}\right\rangle$.

(b) $2 \notin \mathfrak{m}^{2}, \mathfrak{m}^{2}=\left\langle\mathrm{x}^{2}\right\rangle, \mathfrak{m}^{3}=\left\langle 2^{2}\right\rangle=\left\langle\mathrm{x}^{3}\right\rangle$ and $3 \mid 2^{d}-1$.

(28) $\mathrm{GR}\left(2^{3}, d\right)[\mathrm{X}] /\left\langle 2^{2}-\mathrm{X}^{3}, 2 \mathrm{X}\right\rangle$,

(29) $\operatorname{GR}\left(2^{3}, d\right)[\mathrm{X}] /\left\langle 2^{2}-\zeta \mathrm{X}^{3}, 2 \mathrm{X}\right\rangle$,

(30) $\operatorname{GR}\left(2^{3}, d\right)[\mathrm{X}] /\left\langle 2^{2}-\zeta^{2} \mathrm{X}^{3}, 2 \mathrm{X}\right\rangle$.

For the remainder of the manuscript the following notation will be used. Let $(\mathrm{R}, \mathfrak{m}, \operatorname{GF}(q)) \in \mathfrak{F}_{5}^{4}, \mathbb{T}$ and $\mathbb{T}_{s}$ be as above, $\tilde{\alpha}=\left\{\alpha_{1}, \alpha_{2}\right\}$ a minimal Rgenerating set for $\mathfrak{m}, f \in R[\mathrm{~T}]$ a monic basic irreducible polynomial of degree $s$ and $\left(\mathrm{B}=\mathrm{R}[\mathrm{T}] /\langle\mathrm{f}\rangle, \mathfrak{m B}, \mathrm{GF}\left(q^{s}\right)\right)$ the separable extension of $\mathrm{R}$ determined by f.

(1) A fixed minimal R-generating set $\left\{\alpha_{1}, \alpha_{2}\right\}$ of $\mathfrak{m}$ will be considered.

If the ring $\mathrm{R}$ is one of the rings (1) - (13) or (20) - (25) mentioned in Theorem $1, \alpha_{1}=\mathrm{x}$ and $\alpha_{2}=\mathrm{y}$.

If the ring $\mathrm{R}$ is one of the rings (14), (15) mentioned in Theorem 1, $\alpha_{1}=\mathrm{x}$ and $\alpha_{2}=p$. 
SELF DUAL, REVERSIBLE AND COMPLEMENTARY DUALS

CONSTACYCLIC CODES OVER FINITE LOCAL FROBENIUS NON-CHAIN

If the ring $\mathrm{R}$ is one of the rings (16) - (19) mentioned in Theorem 1 , $\alpha_{1}=p$ and $\alpha_{2}=\mathrm{x}$.

If the ring $\mathrm{R}$ is one of the rings (27) - (30) mentioned in Theorem 1 , $\alpha_{1}=2$ and $\alpha_{2}=\mathrm{x}$.

If the ring $\mathrm{R}$ is the ring (26) mentioned in Theorem $1, \alpha_{1}=\mathrm{x}$ and $\alpha_{2}=2$.

When we take a minimal R-generating set for $\mathfrak{m}$ we understand that $\left\{\alpha_{1}, \alpha_{2}\right\}$ is the ordered minimal R-generating set for $\mathfrak{m}$.

(2) Since $\operatorname{ann}_{\mathrm{R}}\left(\mathfrak{m}^{2}\right)=\left\langle\alpha_{1}, \alpha_{2}^{2}\right\rangle,\left\{\alpha_{1}, \alpha_{2}^{2}\right\}$ will be the fixed minimal R-generating set for $\operatorname{ann}_{\mathrm{R}}\left(\mathfrak{m}^{2}\right)$.

When we take a minimal R-generating set for $\operatorname{ann}_{\mathrm{R}}\left(\mathfrak{m}^{2}\right)$ we understand that $\left\{\beta_{1}, \beta_{2}\right\}$ is the ordered minimal R-generating set for $\operatorname{ann}_{\mathrm{R}}\left(\mathfrak{m}^{2}\right)$.

(3) We write $\tilde{\alpha}$ for $\left\{\alpha_{1}, \alpha_{2}\right\}$ and $\tilde{\beta}$ for $\left\{\alpha_{1}, \alpha_{2}^{2}\right\}$.

Lemma 5. Let $(\mathrm{R}, \mathfrak{m}, \operatorname{GF}(q)) \in \mathfrak{F}_{5}^{4}, \mathbb{T}$ and $\mathbb{T}_{s}$ be as above, $\tilde{\alpha}=\left\{\alpha_{1}, \alpha_{2}\right\}$ a minimal $\mathrm{R}$-generating set for $\mathfrak{m}, \mathrm{f} \in \mathrm{R}[\mathrm{T}]$ a monic basic irreducible polynomial of degree $s,\left(\mathrm{~B}=\mathrm{R}[\mathrm{T}] /\langle\mathrm{f}\rangle, \mathfrak{m B}, \mathrm{GF}\left(q^{s}\right)\right)$ the separable extension of $\mathrm{R}$ determined by $\mathrm{f}$, then:

(1) The ideals of length $0,1,4,5$ of $\mathrm{B}$ are $\langle 0\rangle, \mathfrak{m}^{3}, \mathfrak{m}, \mathrm{B}$, respectively.

(2) Ideals of length 2 of $\mathrm{B}$ are between $\operatorname{ann}_{\mathrm{R}}\left(\mathfrak{m}^{2}\right) \mathrm{B}$ and $\mathfrak{m}^{3} \mathrm{~B}$. These ideals are:

$$
\mathfrak{m}^{2}=\left\langle\alpha_{2}^{2}\right\rangle \mathrm{B},\left\langle\alpha_{1}+\lambda_{1}^{\mathbb{T}_{s}} \alpha_{2}^{2}\right\rangle \mathrm{B}, \ldots,\left\langle\alpha_{1}+\lambda_{q^{s}}^{\mathbb{T}_{s}} \alpha_{2}^{2}\right\rangle \mathrm{B}, \quad \lambda_{i} \in \mathrm{GF}\left(q^{s}\right) .
$$

(3) Ideals of length 3 of $\mathrm{B}$ are between $\mathfrak{m B}$ and $\mathfrak{m}^{2} \mathrm{~B}$. These ideals are: $\left\langle\alpha_{1}, \alpha_{2}^{2}\right\rangle \mathrm{B},\left\langle\alpha_{2}\right\rangle \mathrm{B},\left\langle\alpha_{1}+\lambda_{1}^{\mathbb{T}_{s}} \alpha_{2}\right\rangle \mathrm{B}, \ldots,\left\langle\alpha_{1}+\lambda_{q^{s}-1}^{\mathbb{T}_{s}} \alpha_{2}\right\rangle \mathrm{B}, \lambda_{i} \in \mathrm{GF}\left(q^{s}\right) \backslash\{0\}$.

Observation 3. With the notation as in Lemma 5. Observe the following:

(1) $\operatorname{ann}_{\mathrm{B}}(\mathfrak{m B})=\mathfrak{m}^{3} \mathrm{~B}=\left\langle\alpha_{2}^{3}\right\rangle \mathrm{B}=\left\langle\alpha_{1}^{2}\right\rangle \mathrm{B}$.

(2) $\mathfrak{m B}=\left\langle\alpha_{1}, \alpha_{2}\right\rangle \mathrm{B}$ and $\operatorname{ann}_{\mathrm{R}}\left(\mathfrak{m}^{2}\right) \mathrm{B}=\left\langle\alpha_{1}, \alpha_{2}^{2}\right\rangle \mathrm{B}$ are the only non principal ideals of $\mathrm{B}$.

(3) The ideals $(0,1)_{\tilde{\alpha}}^{\mathbb{T}_{s}}=\left\langle\alpha_{2}\right\rangle \mathrm{B},\left(1, \lambda_{i}\right)_{\tilde{\alpha}}^{\mathbb{T}_{s}}=\left\langle\alpha_{1}+\lambda_{i}^{\mathbb{T}_{s}} \alpha_{2}\right\rangle \mathrm{B}, \lambda_{i} \in \mathrm{GF}(q) \backslash\{0\}$, contain $\mathfrak{m}^{2} \mathrm{~B}$.

(4) The ideal $\operatorname{ann}_{\mathrm{R}}\left(\mathfrak{m}^{2}\right) \mathrm{B}=\left\langle\alpha_{1}, \alpha_{2}^{2}\right\rangle \mathrm{B}=(1,0)_{\tilde{\alpha}}^{\mathbb{T}_{s}}+\mathfrak{m}^{2} \mathrm{~B}$ is simply denoted by $(1,0)_{\tilde{\alpha}}^{\mathbb{T} s}$. 
SELF DUAL, REVERSIBLE AND COMPLEMENTARY DUALS

CONSTACYCLIC CODES OVER FINITE LOCAL FROBENIUS NON-CHAIN

RINGS OF LENGTH 5 AND NILPOTENCY INDEX 4

(5) From (1) of Observation 1 and relation $\ell_{\mathrm{B}}(\mathrm{I})+\ell_{\mathrm{B}}\left(\operatorname{ann}_{\mathrm{B}}(\mathrm{I})\right)=\ell_{\mathrm{B}}(\mathrm{B})$, where $\mathrm{I}$ is an ideal of $\mathrm{B}$, the annihilator ideal over the ideals of $\mathrm{B}$ of length 2 and 3 induces the permutations:

$$
\begin{gathered}
(*)^{\perp_{1}},(*)^{\perp_{2}}=\left[(*)^{\perp_{1}}\right]^{-1}:\left\{(0,1),(1, \lambda): \lambda_{i} \in \operatorname{GF}\left(q^{s}\right)\right\} \\
\rightarrow\left\{(0,1),(1, \lambda): \lambda_{i} \in \operatorname{GF}\left(q^{s}\right)\right\} \\
\vec{u} \mapsto \vec{u}^{\perp_{1}}:=\vec{v} \text { if } \operatorname{ann}_{\mathrm{B}}\left(\vec{u}_{\tilde{\alpha}}^{\mathbb{T}_{s}}\right)=\vec{v}_{\tilde{\beta}}^{\mathbb{T}_{s}} \\
\vec{u} \mapsto \vec{u}^{\perp_{2}}:=\vec{v} \text { if } \operatorname{ann}_{\mathrm{B}}\left(\vec{u}_{\tilde{\beta}}^{\mathbb{T}_{s}}\right)=\vec{v}_{\tilde{\alpha}}^{\mathbb{T}_{s}}
\end{gathered}
$$

Lemma 6. Let $(\mathrm{R}, \mathfrak{m}, \mathrm{GF}(q)) \in \mathfrak{F}_{5}^{4}$ be any of the rings (1), (2), (5) - (7), (11), (13), (14), (16), (17), (20) - (28) in Theorem 1. Let $\mathrm{f}, \operatorname{deg}(\mathrm{f})=s$, $\left(\mathrm{B}, \mathfrak{m B}, \operatorname{GF}\left(q^{s}\right)\right), \mathbb{T}, \mathbb{T}_{s}, \tilde{\alpha}=\left\{\alpha_{1}, \alpha_{2}\right\}$ and $\tilde{\beta}=\left\{\alpha_{1}, \alpha_{2}^{2}\right\}$ be as above. Then

$(1,0)^{\perp_{1}}=(0,1), \quad(0,1)^{\perp_{1}}=(1,0), \quad\left(1, \lambda_{1}\right)^{\perp_{1}}=\left(1, \lambda_{2}\right), \lambda_{1}, \lambda_{2} \in \mathbb{T}, \bar{\lambda}_{1} \bar{\lambda}_{2}=-1$

Proof: Assertions follow from the following relations: $\alpha_{1}^{2}=\alpha_{2}^{3}, \alpha_{2} \alpha_{1}=0$, $(0,1)_{\tilde{\alpha}}^{\mathbb{T}_{s}}=\alpha_{2} \mathrm{~B},(1,0)_{\tilde{\beta}}^{\mathbb{T}_{s}}=\alpha_{1} \mathrm{~B},(0,1)_{\tilde{\beta}}^{\mathbb{T}_{s}}=\mathfrak{m}^{2} \mathrm{~B},(1,0)_{\tilde{\alpha}}^{\mathbb{T}_{s}}=\operatorname{ann}_{\mathrm{B}}\left(\mathfrak{m}^{2} \mathrm{~B}\right)$ and $\left(1, \lambda_{1}\right)_{\tilde{\alpha}}^{\mathbb{T}_{s}}\left(1, \lambda_{2}\right)_{\tilde{\beta}}^{\mathbb{T}_{s}}=\left\langle\left(1+\lambda_{1} \lambda_{2}\right) \alpha_{2}^{3}\right\rangle$.

Lemma 7. Let $(\mathrm{R}, \mathfrak{m}, \mathrm{GF}(q)) \in \mathfrak{F}_{5}^{4}$ be any of the rings (3), (4), (8)-(10), (12), (15), (18), (29) in Theorem 1. Let $\mathrm{f}, \operatorname{deg}(\mathrm{f})=s,\left(\mathrm{~B}=\mathrm{R}[\mathrm{T}] /\langle\mathrm{f}\rangle, \mathfrak{m B}, \mathrm{GF}\left(q^{s}\right)\right)$, $\mathbb{T}, \mathbb{T}_{s}, \tilde{\alpha}=\left\{\alpha_{1}, \alpha_{2}\right\}$ and $\tilde{\beta}=\left\{\alpha_{1}, \alpha_{2}^{2}\right\}$ be as above. Then $(1,0)^{\perp_{1}}=(0,1), \quad(0,1)^{\perp_{1}}=(1,0), \quad\left(1, \lambda_{1}\right)^{\perp_{1}}=\left(1, \lambda_{2}\right), \lambda_{1}, \lambda_{2} \in \mathbb{T}, \bar{\lambda}_{1} \bar{\lambda}_{2}=-\bar{\zeta}$.

Proof: Assertions follow from relations $\alpha_{1}^{2}=\zeta \alpha_{2}^{3}$ and $\left(1, \lambda_{1}\right)_{\tilde{\alpha}}^{\mathbb{T}_{s}}\left(1, \lambda_{2}\right)_{\tilde{\beta}}^{\mathbb{T}_{s}}=$ $\left\langle\left(\zeta+\lambda_{1} \lambda_{2}\right) \alpha_{2}^{3}\right\rangle$, and the same kind of arguments as in the proof of Lemma 6 .

Lemma 8. Let $(\mathrm{R}, \mathfrak{m}, \mathrm{GF}(q)) \in \mathfrak{F}_{5}^{4}$ be any of the rings (19), (30) in Theorem 1. Let $\mathrm{f}, \operatorname{deg}(\mathrm{f})=s,\left(\mathrm{~B}=\mathrm{R}[\mathrm{T}] /\langle\mathrm{f}\rangle, \mathfrak{m B}, \mathrm{GF}\left(q^{s}\right)\right), \mathbb{T}, \mathbb{T}_{s}, \tilde{\alpha}=\left\{\alpha_{1}, \alpha_{2}\right\}$ and $\tilde{\beta}=\left\{\alpha_{1}, \alpha_{2}^{2}\right\}$ be as above. Then

$(1,0)^{\perp_{1}}=(0,1), \quad(0,1)^{\perp_{1}}=(1,0), \quad\left(1, \lambda_{1}\right)^{\perp_{1}}=\left(1, \lambda_{2}\right), \lambda_{1}, \lambda_{2} \in \mathbb{T}, \bar{\lambda}_{1} \bar{\lambda}_{2}=-\bar{\zeta}^{2}$.

Proof: Assertions follow from relations $\alpha_{1}^{2}=\zeta^{2} \alpha_{2}^{3}$ and $\left(1, \lambda_{1}\right)_{\tilde{\alpha}}^{\mathbb{T} s}\left(1, \lambda_{2}\right)_{\tilde{\beta}}^{\mathbb{T}_{s}}=$ $\left\langle\left(\zeta^{2}+\lambda_{1} \lambda_{2}\right) \alpha_{2}^{3}\right\rangle$, and the same kind of arguments as in the proof of Lemma 6 .

\section{$3 \quad$ Duals of constacyclic codes over rings in $\mathfrak{F}_{5}^{4}$}

Let $\mathrm{R}$ be a ring and $\gamma$ be a unit of $\mathrm{R}$. A $\gamma$-constacyclic code over $\mathrm{R}$ is a submodule of $\mathrm{R}^{n}$ invariant under the permutation $\sigma_{\gamma}: \mathrm{R}^{n} \rightarrow \mathrm{R}^{n}$ given by $\left(a_{0}, \ldots, a_{n-1}\right) \mapsto\left(\gamma a_{n-1}, a_{0}, \ldots, a_{n-2}\right)$. These codes can be thought as ideals in the ring $\mathrm{R}[\mathrm{T}] /\left\langle\mathrm{T}^{n}-\gamma\right\rangle$ via the $\gamma$-polynomial representation of $\mathrm{R}^{n}, r_{\gamma}: \mathrm{R}^{n} \rightarrow$ 
SELF DUAL, REVERSIBLE AND COMPLEMENTARY DUALS

CONSTACYCLIC CODES OVER FINITE LOCAL FROBENIUS NON-CHAIN

$\mathrm{R}[\mathrm{T}] /\left\langle\mathrm{T}^{n}-\gamma\right\rangle$ given by $\left(a_{0}, \ldots, a_{n-1}\right) \mapsto a_{0}+a_{1} \mathrm{~T}+\ldots+a_{n-1} \mathrm{~T}^{n-1}+\left\langle\mathrm{T}^{n}-\gamma\right\rangle$. The dual of a linear code $\mathrm{C}$ over $\mathrm{R}$ of length $n$ is defined as $\mathrm{C}^{\perp}=\{\vec{a} \in$ $\left.\mathrm{R}^{n}: \vec{a} \cdot \vec{b}=0, \forall \vec{b} \in \mathrm{C}\right\}$, where $\cdot$ is the usual scalar product on $\mathrm{R}^{n}$. Let $\mathrm{f}=\sum_{i=0}^{n-1} a_{i} \mathrm{~T}^{i}+\left\langle\mathrm{T}^{n}-\gamma\right\rangle, \mathrm{g}=\sum_{i=0}^{n-1} b_{i} \mathrm{~T}^{i}+\left\langle\mathrm{T}^{n}-\gamma\right\rangle$ in $\mathrm{R}[\mathrm{T}] /\left\langle\mathrm{T}^{n}-\gamma\right\rangle$, then $\mathrm{fg}^{*}=0$ in $\mathrm{R}[\mathrm{T}] /\left\langle\mathrm{T}^{n}-\gamma\right\rangle$ if and only if $\left(b_{0}, \ldots, b_{n-1}\right)$ is orthogonal to $\left(a_{0}, \ldots, a_{n-1}\right)$ and all of its $\gamma$-constacyclic shifts, if and only if $\left(a_{0}, \ldots, a_{n-1}\right)$ is orthogonal to $\left(b_{0}, \ldots, b_{n-1}\right)$ and all of its $\gamma^{-1}$-constacyclic shifts.

For the rest of this manuscript we write $\sum_{i=0}^{n-1} a_{i} \mathrm{~T}^{i}$ for the corresponding coset $\sum_{i=0}^{n-1} a_{i} \mathrm{~T}^{i}+\left\langle\mathrm{T}^{n}-\gamma\right\rangle$ in the ring $\mathrm{R}[\mathrm{T}] /\left\langle\mathrm{T}^{n}-\gamma\right\rangle, n$ will be the length of codes, $(n, p)=1$, so that, $\mathrm{T}^{n}-\gamma$ is a product of unique monic basic irreducible pairwise coprime polynomials in $\mathrm{R}[\mathrm{T}]$.

The following is the result on the structure of $\gamma$-constacyclic codes over a ring in $\mathfrak{F}_{5}^{4}$, given in [5].

Theorem 2. Let $(\mathrm{R}, \mathfrak{m}, \mathrm{GF}(q)) \in \mathfrak{F}_{5}^{4}, \gamma$ a unit of $\mathrm{R}, \tilde{\alpha}=\left\{\alpha_{1}, \alpha_{2}\right\}, \tilde{\beta}=$ $\left\{\alpha_{1}, \alpha_{2}^{2}\right\}, \mathbb{T}$ and $\mathbb{T}_{s}$ as above and $\mathrm{f}_{1}, \ldots, \mathrm{f}_{r}$ the unique monic basic irreducible pairwise coprime polynomials such that $\mathrm{T}^{n}-\gamma=\mathrm{f}_{1} \cdots \mathrm{f}_{r}, s_{i}=\operatorname{deg}\left(\overline{\mathrm{f}}_{i}\right)$. Let $\mathrm{C}$ a $\gamma$-constacyclic code of length $n$ over $\mathrm{R}$. Then

(1) There exists a unique partition of $[1, r], \mathrm{U}_{0}, \mathrm{U}_{1}, \mathrm{U}_{2}, \mathrm{U}_{3}, \mathrm{U}_{4}, \mathrm{U}_{5}$.

(2) For each $i \in\{2,3\}$ and each $u \in \mathrm{U}_{i}$, there is a unique $\overrightarrow{\mathrm{v}}_{u} \in\{(0,1),(1, \lambda)$ : $\left.\lambda \in \mathrm{GF}\left(q^{s_{u}}\right)\right\}$ such that the corresponding ideal, in $\mathrm{R}[\mathrm{T}] /\left\langle\mathrm{T}^{n}-\gamma\right\rangle$, of $\mathrm{C}$ is

$$
\left\langle\mathfrak{m}^{3} \widehat{\prod_{u \in \mathrm{U}_{1}} \mathrm{f}_{u}}, \widehat{\mathfrak{m}} \widehat{\prod_{u \in \mathrm{U}_{4}} \mathrm{f}_{u}}, \widehat{\prod_{u \in \mathrm{U}_{5}} \mathrm{f}_{u}},\left(\overrightarrow{\mathrm{v}}_{u}\right)_{\tilde{\beta}}^{\mathbb{T}_{s}} \widehat{\mathrm{f}}_{u},\left(\overrightarrow{\mathrm{v}}_{w}\right)_{\tilde{\alpha}}^{\mathbb{T}_{s w}} \widehat{\mathrm{f}}_{w}: u \in \mathrm{U}_{2}, w \in \mathrm{U}_{3}\right\rangle
$$

and

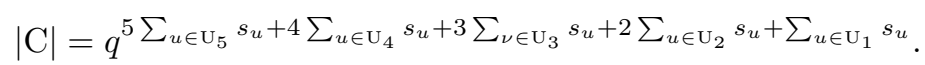

The next result gives the structure of the dual of a constacyclic code over a ring of the family $\mathfrak{F}_{5}^{4}$. Recall that if $\mathrm{C}$ is a linear code of length $n$ over a finite Frobenius ring $\mathrm{R}$, then $|\mathrm{C}|\left|\mathrm{C}^{\perp}\right|=|\mathrm{R}|^{n}$, see [15].

Theorem 3. Let $(\mathrm{R}, \mathfrak{m}, \operatorname{GF}(q)) \in \mathfrak{F}_{5}^{4}, \gamma, \tilde{\alpha}=\left\{\alpha_{1}, \alpha_{2}\right\}, \tilde{\beta}=\left\{\alpha_{1}, \alpha_{2}^{2}\right\}, \mathbb{T}, \mathbb{T}_{s}$, $\mathrm{f}_{1}, \ldots, \mathrm{f}_{r}, s_{i}=\operatorname{deg}\left(\overline{\mathrm{f}}_{i}\right), \mathrm{C}$ as in Theorem 2 and

(1) $\mathrm{U}_{0}, \mathrm{U}_{1}, \mathrm{U}_{2}, \mathrm{U}_{3}, \mathrm{U}_{4}, \mathrm{U}_{5}$ the unique partition of $\{1, \ldots, r\}$ associated to $\mathrm{C}$,

(2) $\left\{\overrightarrow{\mathrm{v}}_{u}: u \in \mathrm{U}_{2} \cup \mathrm{U}_{3}\right\}$ the vectors such that the corresponding ideal, in $\mathrm{R}[\mathrm{T}] /\left\langle\mathrm{T}^{n}-\gamma\right\rangle$, of $\mathrm{C}$ is 
SELF DUAL, REVERSIBLE AND COMPLEMENTARY DUALS

CONSTACYCLIC CODES OVER FINITE LOCAL FROBENIUS NON-CHAIN

RINGS OF LENGTH 5 AND NILPOTENCY INDEX 4

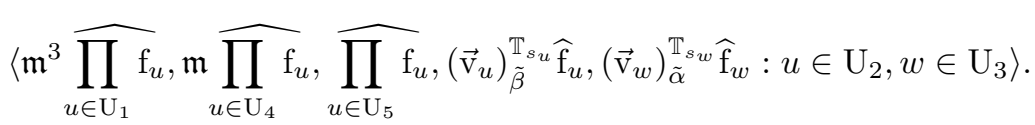

Then

(1) $\mathrm{f}_{1}^{\circ *}, \ldots, \mathrm{f}_{r}^{\circ *}$ are the unique polynomials such that $\mathrm{T}^{n}-\gamma^{-1}=\mathrm{f}_{1}^{\circ *} \cdots \mathrm{f}_{r}^{\circ *}$,

(2) The unique partition of $\{1, \ldots, r\}, \mathrm{V}_{0}, \mathrm{~V}_{1}, \mathrm{~V}_{2}, \mathrm{~V}_{3}, \mathrm{~V}_{4}, \mathrm{~V}_{5}$, associated to the $\gamma^{-1}$ constacyclic code $\mathrm{C}^{\perp}$ is $\mathrm{V}_{0}=\mathrm{U}_{5}, \mathrm{~V}_{1}=\mathrm{U}_{4}, \mathrm{~V}_{2}=\mathrm{U}_{3}, \mathrm{~V}_{3}=\mathrm{U}_{2}, \mathrm{~V}_{4}=\mathrm{U}_{1}$, $\mathrm{V}_{5}=\mathrm{U}_{0}$,

(3) For $u \in \mathrm{V}_{2}$, the unique element in $\{(0,1),(1, \lambda)\}$ over $\mathrm{GF}\left(q^{s_{u}}\right)$ associated to $\mathrm{C}^{\perp}$ is $\overline{\gamma^{-1}}\left(\overrightarrow{\mathrm{v}}_{u}^{\perp_{1}}\right)_{\left(n, \mathrm{f}_{u}^{*}\right)}^{*}, \perp_{1}$ is taken on the ring $\mathrm{R}[\mathrm{T}] /\left\langle\mathrm{f}_{u}\right\rangle$,

(4) For $w \in \mathrm{V}_{3}$, the unique element in $\{(0,1),(1, \lambda)\}$ over $\mathrm{GF}\left(q^{s_{w}}\right)$ associated to $\mathrm{C}^{\perp}$ is $\overline{\gamma^{-1}}\left(\overrightarrow{\mathrm{v}}_{w}^{\perp}\right)_{\left(n, \mathrm{f}_{w}^{*}\right)}^{*}, \perp_{2}$ is taken on the ring $\mathrm{R}[\mathrm{T}] /\left\langle\mathrm{f}_{w}\right\rangle$,

(5) The ideal in $\mathrm{R}[\mathrm{T}] /\left\langle\mathrm{T}^{n}-\gamma^{-1}\right\rangle$, associated to the $\gamma^{-1}$-constacyclic code $\mathrm{C}^{\perp}$ is:

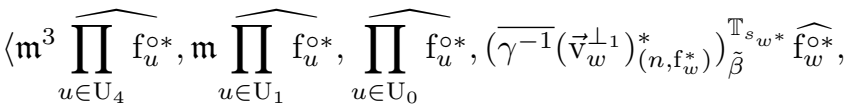

$$
\begin{aligned}
& \left.\left(\overline{\gamma^{-1}}\left(\overrightarrow{\mathrm{v}}_{u}^{\perp_{2}}\right)_{\left(n, \mathrm{f}_{u}^{*}\right)}^{*}\right)_{\tilde{\alpha}}^{\mathbb{T}_{s} u^{*}} \widehat{\mathrm{f}_{u}^{\circ *}}: u \in \mathrm{U}_{2}, w \in \mathrm{U}_{3}\right\rangle= \\
& \left\langle\mathfrak{m}^{3} \widehat{\prod_{u \in \mathrm{U}_{4}} \mathrm{f}_{u^{*}}}, \mathfrak{m} \widehat{\prod_{u \in \mathrm{U}_{1}} \mathrm{f}_{u^{*}}}, \widehat{\prod_{u \in \mathrm{U}_{0}} \mathrm{f}_{u^{*}}},\left(\overline{\gamma^{-1}}\left(\overrightarrow{\mathrm{v}}_{w}^{\perp}\right)_{\left(n, \mathrm{f}_{w^{*}}\right)}^{*}\right)_{\tilde{\beta}}^{\mathbb{T}_{s_{w}}} \widehat{\mathrm{f}_{w^{*}}},\right. \\
& \left.\left(\overline{\gamma^{-1}}\left(\overrightarrow{\mathrm{v}}_{u}^{\perp_{2}}\right)_{\left(n, \mathrm{f}_{u^{*}}\right)}^{*}\right)_{\tilde{\alpha}}^{\mathbb{T}_{s_{u}}} \widehat{\mathrm{f}_{u^{*}}}: u \in \mathrm{U}_{2}, w \in \mathrm{U}_{3}\right\rangle= \\
& \left\langle\mathfrak{m}^{3} \widehat{\prod_{u \in \mathrm{U}_{4}^{*}} \mathrm{f}_{u}}, \mathfrak{m} \widehat{\prod_{u \in \mathrm{U}_{1}^{*}} \mathrm{f}_{u}}, \widehat{\prod_{u \in \mathrm{U}_{0}^{*}} \mathrm{f}_{u}},\left(\overline{\gamma^{-1}}\left(\overrightarrow{\mathrm{v}}_{w^{*}}^{\perp_{1}}\right)_{\left(n, \mathrm{f}_{w}\right)}^{*}\right)_{\tilde{\beta}}^{\mathbb{T}_{s_{w}}} \widehat{\mathrm{f}_{w}},\right. \\
& \left.\left(\overline{\gamma^{-1}}\left(\overrightarrow{\mathrm{v}}_{u^{*}}^{\perp_{2}}\right)_{\left(n, \mathrm{f}_{u}\right)}^{*}\right)_{\tilde{\alpha}}^{\mathbb{T}_{s u}} \widehat{\mathrm{f}_{u}}: u \in \mathrm{U}_{2}^{*}, w \in \mathrm{U}_{3}^{*}\right\rangle
\end{aligned}
$$

and

$$
\left|\mathrm{C}^{\perp}\right|=q^{5 \sum_{u \in \mathrm{U}_{0}} s_{u}+4 \sum_{u \in \mathrm{U}_{1}} s_{u}+3 \sum_{u \in \mathrm{U}_{2}} s_{u}+2 \sum_{u \in \mathrm{U}_{3}} s_{u}+\sum_{u \in \mathrm{U}_{4}} s_{u} .}
$$

Proof: The relation $|\mathrm{M}|=|\mathrm{GF}(q)|^{\ell_{\mathrm{R}}(\mathrm{M})}$ implies $|\mathrm{R}|=q^{5}$, see Section 2 . First, we prove the relation on $\left|\mathrm{C}^{\perp}\right|$. By Theorem 2 and since $n=\sum_{u \in \mathrm{U}_{0}} s_{u}+$ $\sum_{u \in \mathrm{U}_{1}} s_{u}+\sum_{u \in \mathrm{U}_{2}} s_{u}+\sum_{u \in \mathrm{U}_{3}} s_{u}+\sum_{u \in \mathrm{U}_{4}} s_{u}+\sum_{u \in \mathrm{U}_{5}} s_{u}$, then

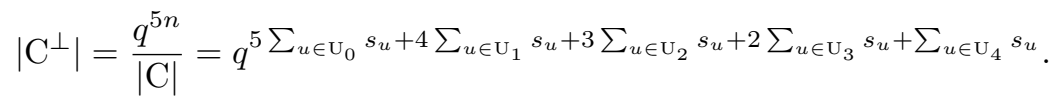

The equality about the ideals is easy to prove. Let $\mathrm{D}$ the $\gamma^{-1}$-constacyclic code given by

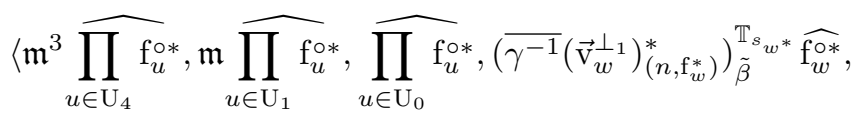


SELF DUAL, REVERSIBLE AND COMPLEMENTARY DUALS

CONSTACYCLIC CODES OVER FINITE LOCAL FROBENIUS NON-CHAIN

RINGS OF LENGTH 5 AND NILPOTENCY INDEX 4

$$
\left.\left(\overline{\gamma^{-1}}\left(\overrightarrow{\mathrm{v}}_{u}^{\perp}\right)_{\left(n, \mathrm{f}_{u}^{*}\right)}^{*}\right)_{\tilde{\alpha}}^{\mathbb{T}_{u^{*}}} \widehat{\mathrm{f}_{u}^{0 *}}: u \in \mathrm{U}_{2}, w \in \mathrm{U}_{3}\right\rangle
$$

Since $\operatorname{deg}\left(\mathrm{f}_{u}^{\circ *}\right)=\operatorname{deg}\left(\mathrm{f}_{u}\right)$, using Theorem 2 , the number of elements of $\mathrm{D}$ is $|\mathrm{D}|=q^{5 \sum_{u \in \mathrm{U}_{0}} s_{u}+4 \sum_{u \in \mathrm{U}_{1}} s_{u}+3 \sum_{u \in \mathrm{U}_{2}} s_{u}+2 \sum_{u \in \mathrm{U}_{3}} s_{u}+\sum_{u \in \mathrm{U}_{4}} s_{u}}=\left|\mathrm{C}^{\perp}\right|$. From (1) of Lemma 3, (1) of Lemma 4 and relations: $\mathfrak{m}^{4}=\langle 0\rangle,\left(\mathrm{f}^{*}\right)^{*}=\mathrm{f}$, when $\mathrm{f}$ is a polynomial with nonzero constant coefficient, $\widehat{\mathrm{f}}_{i} \widehat{\mathrm{f}}_{j}=0$, in the ring $\mathrm{R}[\mathrm{T}] /\left\langle\mathrm{T}^{n}-\right.$ $\gamma\rangle$, for $i \neq j$, we have $\mathrm{D} \subseteq \mathrm{C}^{\perp}$ and the assertion follows.

Let $\mathrm{R}=\mathrm{GF}\left(p^{d}\right)[\mathrm{X}, \mathrm{Y}] /\left\langle\mathrm{X}^{2}-\mathrm{Y}^{3}, \mathrm{XY}\right\rangle$, be the ring of cases (13) or (25) in Theorem 1. Every element of $\mathrm{R}$ can be uniquely written as $a_{0}+a_{1} \mathrm{x}+$ $a_{2} \mathrm{y}+a_{3} \mathrm{y}^{2}+a_{4} \mathrm{y}^{3}$, where $a_{i} \in \mathrm{GF}\left(p^{d}\right), \tilde{\alpha}=\{\mathrm{x}, \mathrm{y}\}, \tilde{\beta}=\left\{\mathrm{x}, \mathrm{y}^{2}\right\}, \mathfrak{m}^{2}=\left\langle\mathrm{y}^{2}\right\rangle$, $\operatorname{ann}_{\mathrm{R}}\left(\mathfrak{m}^{2}\right)=\left\langle\mathrm{x}, \mathrm{y}^{2}\right\rangle, \mathfrak{m}^{3}=\left\langle\mathrm{y}^{3}\right\rangle$ and $\mathbb{T}=\operatorname{GF}\left(p^{d}\right)$ is a set of representatives for the residue field of $R$.

Example 1. Let $\mathrm{R}=\mathrm{GF}(2)[\mathrm{X}, \mathrm{Y}] /\left\langle\mathrm{X}^{2}-\mathrm{Y}^{3}, \mathrm{XY}\right\rangle, \tilde{\alpha}=\{\mathrm{x}, \mathrm{y}\}, \tilde{\beta}=\left\{\mathrm{x}, \mathrm{y}^{2}\right\}$ be as above and $\gamma$ be a unit of $\mathrm{R}$. By Hensel's Lemma, $\mathrm{T}^{15}-\gamma=\mathrm{f}_{1} \mathrm{f}_{2} \mathrm{f}_{3} \mathrm{f}_{4} \mathrm{f}_{5}$, where $\overline{\mathrm{f}_{1}}=\mathrm{T}^{4}+\mathrm{T}+1, \overline{\mathrm{f}_{2}}=\mathrm{T}^{4}+\mathrm{T}^{3}+1, \overline{\mathrm{f}_{3}}=\mathrm{T}^{4}+\mathrm{T}^{3}+\mathrm{T}^{2}+\mathrm{T}+1, \overline{\mathrm{f}_{4}}=\mathrm{T}^{2}+\mathrm{T}+1$, $\overline{\mathrm{f}_{5}}=\mathrm{T}+1 \in \mathrm{GF}(2)[\mathrm{T}]$, then $1^{*}=2,2^{*}=1,3^{*}=3,4^{*}=4,5^{*}=5$.

If $\mathrm{U}_{0}=\{1\}, \mathrm{U}_{1}=\{2\}, \mathrm{U}_{2}=\{3\}, \mathrm{U}_{3}=\{4\}, \mathrm{U}_{4}=\{5\}, \mathrm{U}_{5}=\emptyset$, let $\overrightarrow{\mathrm{v}}_{3}=\left(1,1+\mathrm{T}+\mathrm{T}^{3}\right)$ over $\mathrm{GF}\left(2^{4}\right)=\mathrm{R}[\mathrm{T}] /\left\langle\mathrm{f}_{3}\right\rangle, \overrightarrow{\mathrm{v}}_{4}=(1,1+\mathrm{T})$ over $\mathrm{GF}\left(2^{2}\right)=$ $\mathrm{R}[\mathrm{T}] /\left\langle\mathrm{f}_{4}\right\rangle$. The associated code is:

$$
\begin{gathered}
\left\langle\mathfrak{m}^{3} \widehat{\prod}_{u \in \mathrm{U}_{1}}, \widehat{\mathrm{f}}_{u}, \widehat{\prod}_{u \in \mathrm{U}_{4}}, \widehat{\prod_{u}} \widehat{\mathrm{f}}_{u},\left(\overrightarrow{\mathrm{v}}_{u}\right)_{\tilde{\beta}}^{\mathbb{T}_{s}} \widehat{\mathrm{f}}_{u},\left(\overrightarrow{\mathrm{v}}_{w}\right)_{\tilde{\alpha}}^{\mathbb{T}_{s}} \widehat{\mathrm{f}}_{w}: u \in \mathrm{U}_{2}, w \in \mathrm{U}_{3}\right\rangle= \\
\left\langle\mathrm{y}^{3} \widehat{\mathrm{f}_{2}}, \mathrm{x}_{5}, \widehat{\mathrm{f}}_{5},\left[\mathrm{x}+\left(1+\mathrm{T}+\mathrm{T}^{3}\right) \mathrm{y}^{2}\right] \widehat{\mathrm{f}}_{3},[\mathrm{x}+(1+\mathrm{T}) \mathrm{y}] \widehat{\mathrm{f}}_{4}\right\rangle= \\
\left\langle\mathrm{y}^{3} \mathrm{f}_{1} \mathrm{f}_{3} \mathrm{f}_{4} \mathrm{f}_{5}, \mathrm{xf}_{1} \mathrm{f}_{2} \mathrm{f}_{3} \mathrm{f}_{4}, \mathrm{yf}_{1} \mathrm{f}_{2} \mathrm{f}_{3} \mathrm{f}_{4},\left[\mathrm{x}+\left(1+\mathrm{T}+\mathrm{T}^{3}\right) \mathrm{y}^{2}\right] \mathrm{f}_{1} \mathrm{f}_{2} \mathrm{f}_{4} \mathrm{f}_{5},[\mathrm{x}+(1+\mathrm{T}) \mathrm{y}] \mathrm{f}_{1} \mathrm{f}_{2} \mathrm{f}_{3} \mathrm{f}_{5}\right\rangle .
\end{gathered}
$$

By Lemma 6 and because $\left(1+\mathrm{T}+\mathrm{T}^{3}\right)^{-1}=\mathrm{T}+\mathrm{T}^{2}+\mathrm{T}^{3}$, in $\mathrm{R}[\mathrm{T}] /\left\langle\mathrm{f}_{3}\right\rangle,(1+$ $\mathrm{T})^{-1}=\mathrm{T}$, in $\mathrm{R}[\mathrm{T}] /\left\langle\mathrm{f}_{4}\right\rangle$, we have $\overrightarrow{\mathrm{v}}_{3}^{\perp_{2}}=\left(1, \mathrm{~T}+\mathrm{T}^{2}+\mathrm{T}^{3}\right), \overrightarrow{\mathrm{v}}_{4}^{\perp_{1}}=(1, \mathrm{~T})$. Then $\overline{\gamma^{-1}}\left(\overrightarrow{\mathrm{v}}_{3}^{\perp}\right)_{\left(15, \mathrm{f}_{3}\right)}^{*}=(1,1+\mathrm{T}), \overline{\gamma^{-1}}\left(\overrightarrow{\mathrm{v}}_{4}^{\perp}\right)_{\left(15, \mathrm{f}_{4}\right)}^{*}=(1,1+\mathrm{T})$ and the dual code, $\mathrm{C}^{\perp}$, is:

$$
\begin{gathered}
\left\langle\mathfrak{m}^{3} \widehat{\prod_{u \in \mathrm{U}_{4}} \mathrm{f}_{u}^{*}}, \mathfrak{m} \widehat{\prod_{u \in \mathrm{U}_{1}} \mathrm{f}_{u}^{*}}, \widehat{\prod_{u \in \mathrm{U}_{0}} \mathrm{f}_{u}^{*}}, \overline{\gamma^{-1}}\left(\overrightarrow{\mathrm{v}}_{w}^{\perp_{1}}\right)_{\left(15, \mathrm{f}_{w}^{*}\right)}^{*}\right)_{\tilde{\beta}}^{\mathbb{T}_{s^{*}}} \widehat{\mathrm{f}_{w}^{*}}, \\
\left.\left(\overline{\gamma^{-1}}\left(\overrightarrow{\mathrm{v}}_{u}^{\perp}\right)_{\left(15, \mathrm{f}_{u}^{*}\right)}^{*}\right)_{\tilde{\alpha}}^{\mathbb{T}_{s^{*}}} \widehat{\mathrm{f}_{u}^{*}}: u \in \mathrm{U}_{2}, w \in \mathrm{U}_{3}\right\rangle= \\
\left\langle\mathrm{y}^{3} \widehat{\mathrm{f}_{5}}, \mathrm{xf}_{1}, \mathrm{yf}_{1}, \widehat{\mathrm{f}_{2}},\left[\mathrm{x}+(1+\mathrm{T}) \mathrm{y}^{2}\right] \widehat{\mathrm{f}_{4}},[\mathrm{x}+(1+\mathrm{T}) \mathrm{y}] \widehat{\mathrm{f}_{3}}\right\rangle=\left\langle\mathrm{y}^{3} \mathrm{f}_{1} \mathrm{f}_{2} \mathrm{f}_{3} \mathrm{f}_{4},\right. \\
\left.\mathrm{xf}_{2} \mathrm{f}_{3} \mathrm{f}_{4} \mathrm{f}_{5}, \mathrm{yf}_{2} \mathrm{f}_{3} \mathrm{f}_{4} \mathrm{f}_{5}, \mathrm{f}_{1} \mathrm{f}_{3} \mathrm{f}_{4} \mathrm{f}_{5},\left[\mathrm{x}+(1+\mathrm{T}) \mathrm{y}^{2}\right] \mathrm{f}_{1} \mathrm{f}_{2} \mathrm{f}_{3} \mathrm{f}_{5},[\mathrm{x}+(1+\mathrm{T}) \mathrm{y}] \mathrm{f}_{1} \mathrm{f}_{2} \mathrm{f}_{4} \mathrm{f}_{5}\right\rangle .
\end{gathered}
$$


SELF DUAL, REVERSIBLE AND COMPLEMENTARY DUALS

CONSTACYCLIC CODES OVER FINITE LOCAL FROBENIUS NON-CHAIN

RINGS OF LENGTH 5 AND NILPOTENCY INDEX 4

\section{Self-dual constacyclic codes over rings in $\mathfrak{F}_{5}^{4}$}

The code $\mathrm{C}$ is called self-dual if $\mathrm{C}=\mathrm{C}^{\perp}$. In this Section the algebraic characterization of self-dual $\gamma$-constacyclic codes over rings in the family $\mathfrak{F}_{5}^{4}$ is given, when $\gamma=\gamma^{-1}$.

Let $(\mathrm{R}, \mathfrak{m}, \mathrm{GF}(q))$ be a finite local ring, $\mathrm{f}_{1}, \ldots, \mathrm{f}_{r}$ the unique monic basic irreducible pairwise coprime polynomials such that $\mathrm{T}^{n}-\gamma=\mathrm{f}_{1} \cdots \mathrm{f}_{r}, u \in$ $\{1, \ldots, r\}$ and $\mathrm{U} \subseteq\{1, \ldots, r\}$. Recall that, $\mathrm{U}^{*}$ is the set of indexes of the polynomials which are associates of the polynomials in $\left\{\mathrm{f}_{u}: u \in \mathrm{U}\right\}, u^{*}$ denotes the index of the polynomial which is associate of $\mathrm{f}_{u},\left(u^{*}\right)^{*}=u, \mathrm{f}_{u^{*}}=\mathrm{f}_{u}^{\circ *}$, $\prod_{u \in \mathrm{U}^{\prime}} \mathrm{f}_{u}^{\circ *}=\prod_{u \in \mathrm{U}^{\mathrm{U}}} \mathrm{f}_{u^{*}}=\prod_{u \in \mathrm{U}^{*}} \mathrm{f}_{u}$ and there are $r_{1}, r_{2}$ non negative integers such that $\mathrm{f}_{2 i-1}$ is associate of $\mathrm{f}_{2 i}, 1 \leq i \leq r_{1}$, and $\mathrm{f}_{2 r_{1}+i}$ is self-reciprocal polynomial, $1 \leq i \leq r_{2}$. $\mathrm{U}$ is called self-reciprocal if $\mathrm{U}^{*}=\mathrm{U}$ and special if $\mathrm{U} \cap \mathrm{U}^{*}=\emptyset$.

Theorem 4. Let $(\mathrm{R}, \mathfrak{m}, \operatorname{GF}(q)) \in \mathfrak{F}_{5}^{4}, \gamma, \tilde{\alpha}=\left\{\alpha_{1}, \alpha_{2}\right\}, \tilde{\beta}=\left\{\alpha_{1}, \alpha_{2}^{2}\right\}, \mathbb{T}, \mathbb{T}_{s}$, $\mathrm{f}_{1}, \ldots, \mathrm{f}_{r}, s_{i}=\operatorname{deg}\left(\overline{\mathrm{f}}_{i}\right), \mathrm{C}$ as in Theorem $2, r_{1}, r_{2}$ as above and

(1) $\mathrm{U}_{0}, \mathrm{U}_{1}, \mathrm{U}_{2}, \mathrm{U}_{3}, \mathrm{U}_{4}, \mathrm{U}_{5}$ the unique partition of $\{1, \ldots, r\}$, associated to $\mathrm{C}$,

(2) $\left\{\overrightarrow{\mathrm{v}}_{u}: u \in \mathrm{U}_{2} \cup \mathrm{U}_{3}\right\}$ the vectors such that the corresponding ideal, in $\mathrm{R}[\mathrm{T}] /\left\langle\mathrm{T}^{n}-\gamma\right\rangle$, of $\mathrm{C}$ is

$$
\left\langle\mathfrak{m}^{3} \widehat{\prod_{u \in \mathrm{U}_{1}} \mathrm{f}_{u}}, \mathfrak{m} \widehat{\prod_{u \in \mathrm{U}_{4}} \mathrm{f}_{u}}, \widehat{\prod_{u \in \mathrm{U}_{5}} \mathrm{f}_{u}},\left(\overrightarrow{\mathrm{v}}_{u}\right)_{\tilde{\beta}^{\prime}}^{\mathbb{T}_{s}} \widehat{\mathrm{f}}_{u},\left(\overrightarrow{\mathrm{v}}_{w}\right)_{\tilde{\alpha}}^{\mathbb{T}_{s}} \widehat{\mathrm{f}}_{w}: u \in \mathrm{U}_{2}, w \in \mathrm{U}_{3}\right\rangle .
$$

The following conditions are equivalent:

(1) C is self-dual code;

(2) $\mathrm{U}_{i}^{*}=\mathrm{U}_{5-i}$, for $i \in\{0, \ldots, 5\}, \overrightarrow{\mathrm{v}}_{u}=\bar{\gamma}\left(\overrightarrow{\mathrm{v}}_{u^{*}}^{\perp_{1}}\right)_{\left(n, \mathrm{f}_{u}\right)}^{*}$, for $u \in \mathrm{U}_{2}$ and $\overrightarrow{\mathrm{v}}_{w}=\bar{\gamma}\left(\overrightarrow{\mathrm{v}}_{w^{*}}^{\perp}\right)_{\left(n, \mathrm{f}_{w}\right)}^{*}$, for $w \in \mathrm{U}_{3}$.

(3) $\mathrm{U}_{i}^{*} \subseteq \mathrm{U}_{5-i}$, for $i \in\{0, \ldots, 5\}, \overrightarrow{\mathrm{v}}_{u}=\bar{\gamma}\left(\overrightarrow{\mathrm{v}}_{u^{*}}^{\perp_{1}}\right)_{\left(n, \mathrm{f}_{u}\right)}^{*}$, for $u \in \mathrm{U}_{2}$ and $\overrightarrow{\mathrm{v}}_{w}=\bar{\gamma}\left(\overrightarrow{\mathrm{v}}_{w^{*}}^{+}\right)_{\left(n, \mathrm{f}_{w}\right)}^{*}$, for $w \in \mathrm{U}_{3}$.

Proof: $(2) \Leftrightarrow(3)$ is easy to prove.

$(1) \Leftrightarrow(2)$ From uniqueness in Theorem 2 , by Theorem 3 and since $\left\{U_{0}^{*}, U_{1}^{*}\right.$, $\left.\mathrm{U}_{2}^{*}, \mathrm{U}_{3}^{*}, \mathrm{U}_{4}^{*}, \mathrm{U}_{5}^{*}\right\}$ is a partition of $\{1, \ldots, r\}$,

$\mathrm{C}=\mathrm{C}^{\perp} \Leftrightarrow$

$$
\left\langle\mathfrak{m}^{3} \widehat{\prod_{u \in \mathrm{U}_{1}} \mathrm{f}_{u}}, \mathfrak{m} \widehat{\prod_{u \in \mathrm{U}_{4}} \mathrm{f}_{u}}, \widehat{\prod_{u \in \mathrm{U}_{5}} \mathrm{f}_{u}},\left(\overrightarrow{\mathrm{v}}_{u}\right)_{\tilde{\beta}}^{\mathbb{T}_{s_{u}}} \widehat{\mathrm{f}}_{u},\left(\overrightarrow{\mathrm{v}}_{w}\right)_{\tilde{\alpha}}^{\mathbb{T}_{s}} \widehat{\mathrm{f}}_{w}: u \in \mathrm{U}_{2}, w \in \mathrm{U}_{3}\right\rangle=
$$


SELF DUAL, REVERSIBLE AND COMPLEMENTARY DUALS

CONSTACYCLIC CODES OVER FINITE LOCAL FROBENIUS NON-CHAIN

RINGS OF LENGTH 5 AND NILPOTENCY INDEX 4

$$
\begin{aligned}
& \left\langle\mathfrak{m}^{3} \widehat{\prod_{u \in \mathrm{U}_{4}^{*}} \mathrm{f}_{u}}, \mathfrak{m} \widehat{\prod_{u \in \mathrm{U}_{1}^{*}} \mathrm{f}_{u}}, \widehat{\prod_{u \in \mathrm{U}_{0}^{*}} \mathrm{f}_{u}},\left(\bar{\gamma}\left(\overrightarrow{\mathrm{v}}_{w^{*}}^{\perp_{1}}\right)_{\left(n, \mathrm{f}_{w}\right)}^{*}\right)_{\tilde{\beta}}^{\mathbb{T}_{s_{w}}} \widehat{\mathrm{f}_{w}},\left(\bar{\gamma}\left(\overrightarrow{\mathrm{v}}_{u^{*}}^{\perp_{2}}\right)_{\left(n, \mathrm{f}_{u}\right)}^{*}\right)_{\tilde{\alpha}}^{\mathbb{T}_{s_{u}}} \widehat{\mathrm{f}_{u}}:\right. \\
& \left.u \in \mathrm{U}_{2}^{*}, w \in \mathrm{U}_{3}^{*}\right\rangle,
\end{aligned}
$$

$\Leftrightarrow \mathrm{U}_{1}=\mathrm{U}_{4}^{*}, \mathrm{U}_{5}=\mathrm{U}_{0}^{*}, \mathrm{U}_{2}=\mathrm{U}_{3}^{*},\left\langle\left(\overrightarrow{\mathrm{v}}_{u}\right)_{\tilde{\beta}}^{\mathbb{T}_{s}} \widehat{\mathrm{f}}_{u}: u \in \mathrm{U}_{2}\right\rangle=\left\langle\left(\bar{\gamma}\left(\overrightarrow{\mathrm{v}}_{u^{*}}^{\perp_{1}}\right)_{\left(n, \mathrm{f}_{u}\right)}^{*}\right)_{\tilde{\beta}}^{\mathbb{T}_{s_{u}}} \widehat{\mathrm{f}_{u}}:\right.$ $\left.u \in \mathrm{U}_{3}^{*}\right\rangle$ and $\left.\left\langle\left(\overrightarrow{\mathrm{v}}_{u}\right)_{\tilde{\alpha}}^{\mathbb{T}_{s}} \widehat{\mathrm{f}}_{u}: u \in \mathrm{U}_{3}\right\rangle=\left(\bar{\gamma}\left(\overrightarrow{\mathrm{v}}_{u^{*}}^{\perp}\right)_{\left(n, \mathrm{f}_{u}\right)}^{*}\right)_{\tilde{\alpha}}^{\mathbb{T}_{s}} \widehat{\mathrm{f}_{u}}: u \in \mathrm{U}_{2}^{*}\right\rangle$,

$\Leftrightarrow \mathrm{U}_{1}=\mathrm{U}_{4}^{*}, \mathrm{U}_{5}=\mathrm{U}_{0}^{*}, \mathrm{U}_{2}=\mathrm{U}_{3}^{*}$, for each $u \in \mathrm{U}_{2}$ there is $\varepsilon \in \mathrm{U}_{3}$, for each

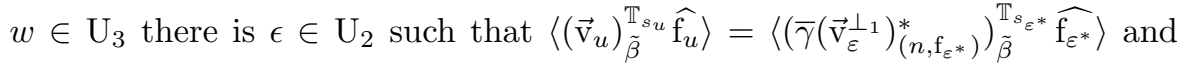
$\left\langle\left(\overrightarrow{\mathrm{v}}_{w}\right)_{\tilde{\alpha}}^{\mathbb{T}_{s w}} \widehat{\mathrm{f}_{w}}\right\rangle=\left\langle\left(\bar{\gamma}\left(\overrightarrow{\mathrm{v}}_{\epsilon}^{\perp}\right)_{\left(n, \mathrm{f}_{\epsilon^{*}}\right)}^{*}\right)_{\tilde{\alpha}}^{\mathbb{T}_{\epsilon^{*}}} \widehat{\mathrm{f}_{\epsilon^{*}}}\right\rangle$, $\Leftrightarrow \mathrm{U}_{1}=\mathrm{U}_{4}^{*}, \mathrm{U}_{5}=\mathrm{U}_{0}^{*}, \mathrm{U}_{2}=\mathrm{U}_{3}^{*}$, for each $u \in \mathrm{U}_{2}$ there is $\varepsilon \in \mathrm{U}_{3}$, for each $w \in \mathrm{U}_{3}$ there is $\epsilon \in \mathrm{U}_{2}$ such that $\mathrm{f}_{u}=\mathrm{f}_{\varepsilon^{*}}, \mathrm{f}_{w}=\mathrm{f}_{\epsilon^{*}}, u=\varepsilon^{*}, w=\epsilon^{*}$, $\overrightarrow{\mathrm{v}}_{u}=\bar{\gamma}\left(\overrightarrow{\mathrm{v}}_{u^{*}}^{\perp_{1}}\right)_{\left(n, \mathrm{f}_{u}\right)}^{*}$ and $\overrightarrow{\mathrm{v}}_{w}=\bar{\gamma}\left(\overrightarrow{\mathrm{v}}_{w^{*}}^{\perp_{2}}\right)_{\left(n, \mathrm{f}_{w}\right)}^{*}$, by Lemma 5 .

For the next result recall that if $\bar{\gamma}=1$, the binomial $\mathrm{T}^{n}-\gamma$ always has a basic irreducible self reciprocal factor in $\mathrm{R}[\mathrm{T}]$ and if $\bar{\gamma}=-1$, the binomial $\mathrm{T}^{n}-\gamma$ might not have a basic irreducible self reciprocal factor in $\mathrm{R}[\mathrm{T}]$.

Corollary 1. With the notation as in Theorem 4, if $\mathrm{C}$ is a self-dual code there is not $u \in\{1, \ldots, r\}$ such that $\mathrm{f}_{u}$ is self reciprocal.

In particular if $\bar{\gamma}=1$ there is not any self dual $\gamma$-constacyclic code over $\mathrm{R}$.

Proof: Since $\mathrm{U}_{1}=\mathrm{U}_{4}^{*}, \mathrm{U}_{5}=\mathrm{U}_{0}^{*}$ and $\mathrm{U}_{2}=\mathrm{U}_{3}^{*}$, then each $\mathrm{U}_{i}$ is special and the assertion follows.

In the following lines some examples are given illustrating the above results.

Example 2. Let $\left(\mathrm{R}, \mathfrak{m}, \mathrm{GF}\left(2^{d}\right)\right) \in \mathfrak{F}_{5}^{4}$ and $\gamma$ a unit of R. From Corollary 1, there is not any self dual $\gamma$-constacyclic code over $\mathrm{R}$.

Example 3. Let $\mathrm{R}=\mathrm{GF}(3)[\mathrm{X}, \mathrm{Y}] /\left\langle\mathrm{X}^{2}-\mathrm{Y}^{3}, \mathrm{XY}\right\rangle$ be the ring of case (13) in Theorem 1, $\tilde{\alpha}=\{\mathrm{x}, \mathrm{y}\}, \tilde{\beta}=\left\{\mathrm{x}, \mathrm{y}^{2}\right\}$ as above and $\gamma=2$. By Hensel's Lemma, $\mathrm{T}^{4}-2=\mathrm{T}^{4}+1=\mathrm{f}_{1} \mathrm{f}_{2}$, where $\mathrm{f}_{1}=\mathrm{T}^{2}+2 \mathrm{~T}+2, \mathrm{f}_{2}=\mathrm{T}^{2}+\mathrm{T}+2 \in \mathrm{R}[\mathrm{T}]$. We have $1^{*}=2$.

Let $\mathrm{U}_{0}=\mathrm{U}_{1}=\mathrm{U}_{4}=\mathrm{U}_{5}=\emptyset, \mathrm{U}_{2}=\{1\}, \mathrm{U}_{3}=\{2\}, \overrightarrow{\mathrm{v}}_{1}=(1,1+\mathrm{T})$ over $\mathrm{GF}\left(2^{2}\right)=\mathrm{R}[\mathrm{T}] /\left\langle\mathrm{f}_{1}\right\rangle, \overrightarrow{\mathrm{v}}_{1}^{\perp_{2}}=(1,1+\mathrm{T})$, by Lemma $6, \overrightarrow{\mathrm{v}}_{2}=2\left(\overrightarrow{\mathrm{v}}_{1}^{\perp_{2}}\right)_{\left(4, \mathrm{f}_{2}\right)}^{*}=$ $(1,2+\mathrm{T})$, over $\mathrm{GF}\left(2^{2}\right)=\mathrm{R}[\mathrm{T}] /\left\langle\mathrm{f}_{2}\right\rangle$.

Then the $\gamma$-constacyclic code

$$
\begin{aligned}
& \mathrm{C}=\left\langle\mathrm{m}^{3} \widehat{\prod_{u \in \mathrm{U}_{1}} \mathrm{f}_{u}}, \mathfrak{m} \widehat{\prod_{u \in \mathrm{U}_{4}} \mathrm{f}_{u}}, \widehat{\prod_{u \in \mathrm{U}_{5}} \mathrm{f}_{u}},\left(\overrightarrow{\mathrm{v}}_{u}\right)_{\tilde{\beta}}^{\left.\mathbb{T}_{\tilde{s}_{u}} \widehat{\mathrm{f}}_{u},\left(\overrightarrow{\mathrm{v}}_{w}\right)_{\tilde{\alpha}}^{\mathbb{T}_{s_{w}} \widehat{\mathrm{f}}_{w}}: u \in \mathrm{U}_{2}, w \in \mathrm{U}_{3}\right\rangle=}\right. \\
& \left\langle\left[\mathrm{x}+(1+\mathrm{T}) \mathrm{y}^{2}\right] \widehat{\mathrm{f}}_{1},[\mathrm{x}+(2+\mathrm{T}) \mathrm{y}] \widehat{\mathrm{f}}_{2}\right\rangle=\left\langle\left[\mathrm{x}+(1+\mathrm{T}) \mathrm{y}^{2}\right] \mathrm{f}_{2},[\mathrm{x}+(2+\mathrm{T}) \mathrm{y}] \mathrm{f}_{1}\right\rangle
\end{aligned}
$$

is self-dual. 
SELF DUAL, REVERSIBLE AND COMPLEMENTARY DUALS

CONSTACYCLIC CODES OVER FINITE LOCAL FROBENIUS NON-CHAIN

RINGS OF LENGTH 5 AND NILPOTENCY INDEX 4

\section{Reversible constacyclic codes over rings in $\mathfrak{F}_{5}^{4}$}

A linear code $\mathrm{C}$ of length $n$ over the ring $\mathrm{R}$ is reversible if it is invariant under the reversible permutation $\mathfrak{r}: \mathrm{R}^{n} \rightarrow \mathrm{R}^{n}$ given by $\left(a_{0}, a_{1}, \ldots, a_{n-1}\right) \mapsto$ $\left(a_{n-1}, \ldots, a_{1}, a_{0}\right)$. In this Section the algebraic characterization of reversible $\gamma$-constacyclic codes over rings in the family $\mathfrak{F}_{5}^{4}$ is given, when $\gamma=\gamma^{-1}$.

The following result will be useful; claim (2) was treated in [4].

Lemma 9. Let $\mathrm{R}$ be a finite ring, $\gamma$ a unit of $\mathrm{R}, \mathfrak{r}, \sigma_{\gamma}$ and $r_{\gamma}$ the reversible permutation, the $\gamma$-constacyclic permutation and the $\gamma$-polynomial representation of $\mathrm{R}^{n}$, respectively, then:

(1) $\mathfrak{r} \circ \sigma_{\gamma}^{n-1}(\gamma \vec{a})=\sigma_{\gamma} \circ \mathfrak{r}(\vec{a})$, for all $\vec{a} \in \mathrm{R}^{n}$.

(2) $\gamma \mathrm{f}^{*}=r_{\gamma} \circ \sigma_{\gamma}^{k+1} \circ \mathfrak{r} \circ r_{\gamma}^{-1}(\mathrm{f})$, for $\mathrm{f}=a_{0}+\ldots+a_{n-1} \mathrm{~T}^{n-1} \in \mathrm{R}[\mathrm{T}] /\left\langle\mathrm{T}^{n}-\gamma\right\rangle$ with $\operatorname{deg}(\mathrm{f})=k$.

Proof: (1) Let $\vec{a}=\left(a_{0}, a_{1}, \ldots, a_{n-1}\right)$, then

$$
\begin{gathered}
\mathfrak{r} \circ \sigma_{\gamma} \circ \mathfrak{r}(\vec{a})=\mathfrak{r} \circ \sigma_{\gamma}\left(a_{n-1}, \ldots, a_{1}, a_{0}\right)=\mathfrak{r}\left(\gamma a_{0}, a_{n-1}, \ldots, a_{1}\right)= \\
\left(a_{1}, \ldots, a_{n-1}, \gamma a_{0}\right)=\sigma_{\gamma}^{n-1}\left(\gamma\left(a_{0}, a_{1}, \ldots, a_{n-1}\right)\right)=\sigma_{\gamma}^{n-1}(\gamma(\vec{a})) .
\end{gathered}
$$

Observation 4. Under the notation as in Lemma 9, let $\mathrm{C}$ be a $\gamma$-constacyclic code.

(1) From relation (1) of Lemma 9, $\sigma_{\gamma}(\mathfrak{r}(\mathrm{C}))=\mathfrak{r} \circ \sigma_{\gamma}^{n-1}(\gamma \mathrm{C})=\mathfrak{r} \circ \sigma_{\gamma}^{n-1}(\mathrm{C})=$ $\mathfrak{r}(\mathrm{C})$ and $\mathfrak{r}(\mathrm{C})$ is a $\gamma$-constacyclic code.

(2) Let $\vec{a} \in \mathrm{R}^{n}$, relation $\sigma_{\gamma}^{n}(\vec{a})=\gamma \vec{a}$ and (2) of Lemma 9, imply $\sigma_{\gamma}^{n-k-1}$ 。 $r_{\gamma}^{-1}\left[\left(r_{\gamma}(\vec{a})\right)^{*}\right]=\mathfrak{r}(\vec{a})$, where $k=\operatorname{deg}\left(r_{\gamma}(\vec{a})\right)$. This relation means that the reversible permutation of $\vec{a}$ can be obtained from the $\gamma$-constacyclic permutation and the reciprocal operation of $r_{\gamma}(\vec{a})$. This implies that the ideal corresponding to $\mathfrak{r}(\mathrm{C})$ is the ideal generated by $\left\{\mathrm{f}^{*}: \mathrm{f} \in r_{\gamma}(\mathrm{C})\right\}$.

For the next result recall that if $\delta_{1}, \ldots, \delta_{k}, \mathrm{~h}_{1}, \ldots, \mathrm{h}_{k} \in \mathrm{R}[\mathrm{T}]$ and the leader coefficient of each $\delta_{i}$ is a unit, then $\left(\delta_{1} \mathrm{~h}_{1}+\ldots+\delta_{k} \mathrm{~h}_{k}\right)^{*}=\mathrm{T}^{u_{1}} \delta_{1}^{*} \mathrm{~h}_{1}^{*}+\ldots+$ $\mathrm{T}^{u_{k}} \delta_{k}^{*} \mathrm{~h}_{1}^{*}$, for some $u_{1}, \ldots, u_{k}$ non negative integers, see Section 2 . This implies if $\psi \in\left\langle\delta_{1}, \ldots, \delta_{k}\right\rangle$ then $\psi^{*} \in\left\langle\delta_{1}^{*}, \ldots, \delta_{k}^{*}\right\rangle$.

Lemma 10. Let $(\mathrm{R}, \mathfrak{m}, \operatorname{GF}(q)) \in \mathfrak{F}_{5}^{4}, \gamma, \tilde{\alpha}=\left\{\alpha_{1}, \alpha_{2}\right\}, \tilde{\beta}=\left\{\alpha_{1}, \alpha_{2}^{2}\right\}, \mathbb{T}, \mathbb{T}_{s}$, $\mathrm{f}_{1}, \ldots, \mathrm{f}_{r}, s_{i}=\operatorname{deg}\left(\overline{\mathrm{f}}_{i}\right), \mathrm{C}$ as in Theorem $2, \mathfrak{r}$ the reversible permutation on $\mathrm{R}^{n}$ and

(1) $\mathrm{U}_{0}, \mathrm{U}_{1}, \mathrm{U}_{2}, \mathrm{U}_{3}, \mathrm{U}_{4}, \mathrm{U}_{5}$ the unique partition of $\{1, \ldots, r\}$, associated to $\mathrm{C}$, 
SELF DUAL, REVERSIBLE AND COMPLEMENTARY DUALS

CONSTACYCLIC CODES OVER FINITE LOCAL FROBENIUS NON-CHAIN

(2) $\left\{\overrightarrow{\mathrm{v}}_{u}: u \in \mathrm{U}_{2} \cup \mathrm{U}_{3}\right\}$ the vectors such that the corresponding ideal, in $\mathrm{R}[\mathrm{T}] /\left\langle\mathrm{T}^{n}-\gamma\right\rangle$, of $\mathrm{C}$ is

$$
\left\langle\mathfrak{m}^{3} \widehat{\prod_{u \in \mathrm{U}_{1}} \mathrm{f}_{u}}, \mathfrak{m} \widehat{\prod_{u \in \mathrm{U}_{4}} \mathrm{f}_{u}}, \widehat{\prod_{u \in \mathrm{U}_{5}} \mathrm{f}_{u}},\left(\overrightarrow{\mathrm{v}}_{u}\right)_{\tilde{\beta}}^{\mathbb{T}_{s_{u}}} \widehat{\mathrm{f}}_{u},\left(\overrightarrow{\mathrm{v}}_{w}\right)_{\tilde{\alpha}}^{\mathbb{T}_{s_{w}}} \widehat{\mathrm{f}}_{w}: u \in \mathrm{U}_{2}, w \in \mathrm{U}_{3}\right\rangle .
$$

Then

(1) $\mathrm{V}_{0}=\mathrm{U}_{0}^{*}, \mathrm{~V}_{1}=\mathrm{U}_{1}^{*}, \mathrm{~V}_{2}=\mathrm{U}_{2}^{*}, \mathrm{~V}_{3}=\mathrm{U}_{3}^{*}, \mathrm{~V}_{4}=\mathrm{U}_{4}^{*}, \mathrm{~V}_{5}=\mathrm{U}_{5}^{*}$ is the unique partition of $\{1, \ldots, r\}$, associated to $\mathfrak{r}(\mathrm{C})$,

(2) $\left\{\bar{\gamma}\left(\overrightarrow{\mathrm{v}}_{u^{*}}\right)_{\left(n, \mathrm{f}_{u}\right)}^{*}, \bar{\gamma}\left(\overrightarrow{\mathrm{v}}_{w^{*}}\right)_{\left(n, \mathrm{f}_{w}\right)}^{*}: u \in \mathrm{V}_{2}=\mathrm{U}_{2}^{*}, w \in \mathrm{V}_{3}=\mathrm{U}_{3}^{*}\right\}$ are the unique vectors such that the corresponding ideal, in $\mathrm{R}[\mathrm{T}] /\left\langle\mathrm{T}^{n}-\gamma\right\rangle$, of $\mathfrak{r}(\mathrm{C})$ is

$$
\begin{gathered}
\left\langle\mathfrak{m}^{3} \widehat{\prod_{u \in \mathrm{U}_{1}^{*}} \mathrm{f}_{u}}, \mathfrak{m} \widehat{\prod_{u \in \mathrm{U}_{4}^{*}} \mathrm{f}_{u}}, \widehat{\prod_{u \in \mathrm{U}_{5}^{*}} \mathrm{f}_{u}},\left(\bar{\gamma}\left(\overrightarrow{\mathrm{v}}_{u^{*}}\right)_{\left(n, \mathrm{f}_{u}\right)}^{*}\right)_{\tilde{\beta}}^{\mathbb{T}_{s_{u}}} \widehat{\mathrm{f}_{u}},\left(\bar{\gamma}\left(\overrightarrow{\mathrm{v}}_{w^{*}}\right)_{\left(n, \mathrm{f}_{w}\right)}^{*}\right)_{\tilde{\alpha}}^{\mathbb{T}_{s_{w}}} \widehat{\mathrm{f}_{w}}:\right. \\
\left.u \in \mathrm{U}_{2}^{*}, w \in \mathrm{U}_{3}^{*}\right\rangle .
\end{gathered}
$$

Proof: Let $\mathrm{D}$ the $\gamma$-constacyclic code given by

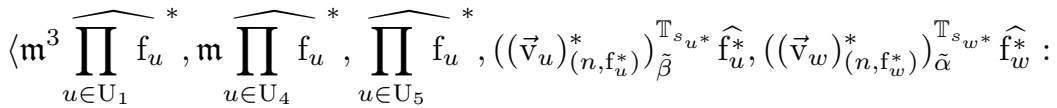

$$
\begin{aligned}
& \left.u \in \mathrm{U}_{2}, w \in \mathrm{U}_{3}\right\rangle= \\
& \left\langle\mathfrak{m}^{3} \widehat{\prod_{u \in \mathrm{U}_{1}^{*}} \mathrm{f}_{u}}, \mathfrak{m} \widehat{\prod_{u \in \mathrm{U}_{4}^{*}} \mathrm{f}_{u}}, \widehat{\prod_{u \in \mathrm{U}_{5}^{*}} \mathrm{f}_{u}},\left(\bar{\gamma}\left(\overrightarrow{\mathrm{v}}_{u^{*}}\right)_{\left(n, \mathrm{f}_{u^{*}}^{*}\right)}^{*}\right)_{\tilde{\beta}}^{\mathbb{T}_{s}\left(u^{*}\right)^{*}} \widehat{\mathrm{f}_{u^{*}}^{*}},\right. \\
& \left.\left(\bar{\gamma}\left(\overrightarrow{\mathrm{v}}_{w^{*}}\right)_{\left(n, \mathrm{f}_{w^{*}}^{*}\right)}^{*}\right)_{\tilde{\alpha}}^{\mathbb{T}_{s}}{ }_{\left(w^{*}\right)^{*}} \mathrm{f}_{w^{*}}^{*}: u \in \mathrm{U}_{2}^{*}, w \in \mathrm{U}_{3}^{*}\right\rangle= \\
& \left\langle\mathfrak{m}^{3} \widehat{\prod_{u \in \mathrm{U}_{1}^{*}} \mathrm{f}_{u}}, \widehat{\mathfrak{m}} \widehat{\prod_{u \in \mathrm{U}_{4}^{*}} \mathrm{f}_{u}}, \widehat{\prod_{u \in \mathrm{U}_{5}^{*}} \mathrm{f}_{u}},\left(\bar{\gamma}\left(\overrightarrow{\mathrm{v}}_{u^{*}}\right)_{\left(n, \mathrm{f}_{u}\right)}^{*}\right)_{\tilde{\beta}}^{\mathbb{T}_{s_{u}}} \widehat{\mathrm{f}_{u}},\left(\bar{\gamma}\left(\overrightarrow{\mathrm{v}}_{w^{*}}\right)_{\left(n, \mathrm{f}_{w}\right)}^{*}\right)_{\tilde{\alpha}}^{\mathbb{T}_{s_{w}}} \widehat{\mathrm{f}_{w}}:\right. \\
& \left.u \in \mathrm{U}_{2}^{*}, w \in \mathrm{U}_{3}^{*}\right\rangle .
\end{aligned}
$$

By (2) and (3) of Lemma 3, (2) and (3) of Lemma 4, and Observation 4, the corresponding ideal of $\mathfrak{r}(\mathrm{C})$ is contained in D. Since $\operatorname{deg}\left(\mathrm{f}_{i}\right)=\operatorname{deg}\left(\mathrm{f}_{i}^{*}\right)$, using Theorem 2, the number of elements in $\mathrm{D}$ and in $\mathrm{C}$ is the same. The assertion follows.

Theorem 5. Let $(\mathrm{R}, \mathfrak{m}, \operatorname{GF}(q)) \in \mathfrak{F}_{5}^{4}, \gamma, \tilde{\alpha}=\left\{\alpha_{1}, \alpha_{2}\right\}, \tilde{\beta}=\left\{\alpha_{1}, \alpha_{2}^{2}\right\}, \mathbb{T}, \mathbb{T}_{s}$, $\mathrm{f}_{1}, \ldots, \mathrm{f}_{r}$ and $s_{i}=\operatorname{deg}\left(\overline{\mathrm{f}}_{i}\right), \mathrm{C}$ as in Theorem 2 and

(1) $\mathrm{U}_{0}, \mathrm{U}_{1}, \mathrm{U}_{2}, \mathrm{U}_{3}, \mathrm{U}_{4}, \mathrm{U}_{5}$ the unique partition of $\{1, \ldots, r\}$, associated to $\mathrm{C}$, 
SELF DUAL, REVERSIBLE AND COMPLEMENTARY DUALS

CONSTACYCLIC CODES OVER FINITE LOCAL FROBENIUS NON-CHAIN

(2) $\left\{\overrightarrow{\mathrm{v}}_{u}: u \in \mathrm{U}_{2} \cup \mathrm{U}_{3}\right\}$ the vectors such that the corresponding ideal, in $\mathrm{R}[\mathrm{T}] /\left\langle\mathrm{T}^{n}-\gamma\right\rangle$, of $\mathrm{C}$ is

$$
\left\langle\mathfrak{m}^{3} \widehat{\prod_{u \in \mathrm{U}_{1}} \mathrm{f}_{u}}, \mathfrak{m} \widehat{\prod_{u \in \mathrm{U}_{4}} \mathrm{f}_{u}}, \widehat{\prod_{u \in \mathrm{U}_{5}} \mathrm{f}_{u}},\left(\overrightarrow{\mathrm{v}}_{u}\right)_{\tilde{\beta}}^{\mathbb{T}_{s_{u}}} \widehat{\mathrm{f}}_{u},\left(\overrightarrow{\mathrm{v}}_{w}\right)_{\tilde{\alpha}}^{\mathbb{T}_{s_{w}}} \widehat{\mathrm{f}}_{w}: u \in \mathrm{U}_{2}, w \in \mathrm{U}_{3}\right\rangle .
$$

The following conditions are equivalent:

(1) $\mathrm{C}$ is a reversible code;

(2) $\mathrm{U}_{i}$ is self-reciprocal, for $i \in\{1, \ldots, 5\}$, and $\overrightarrow{\mathrm{v}}_{u}=\bar{\gamma}\left(\overrightarrow{\mathrm{v}}_{u^{*}}\right)_{\left(n, \mathrm{f}_{u}\right)}^{*}$, for $u \in$ $\mathrm{U}_{2} \cup \mathrm{U}_{3}$;

(3) $\mathrm{U}_{i}$ is self-reciprocal, for $i \in\{0, \ldots, 5\}$, and $\overrightarrow{\mathrm{v}}_{u}=\bar{\gamma}\left(\overrightarrow{\mathrm{v}}_{u^{*}}\right)_{\left(n, \mathrm{f}_{u}\right)}^{*}$, for $u \in$ $\mathrm{U}_{2} \cup \mathrm{U}_{3}$.

Proof: $(1) \Leftrightarrow(2) \mathrm{C}$ is reversible $\Leftrightarrow$ $\mathrm{U}_{1}=\mathrm{U}_{1}^{*}, \mathrm{U}_{4}=\mathrm{U}_{4}^{*}, \mathrm{U}_{5}=\mathrm{U}_{5}^{*}$, for each $u \in \mathrm{U}_{2}$ there exists $\varepsilon \in \mathrm{U}_{2}^{*}$, for each $w \in \mathrm{U}_{3}$ there exists $\epsilon \in \mathrm{U}_{3}^{*}$ such that $\left\langle\left(\overrightarrow{\mathrm{v}}_{u}\right)_{\tilde{\beta}}^{\mathbb{T}_{s_{u}}} \widehat{\mathrm{f}_{u}}\right\rangle=\left\langle\left(\bar{\gamma}\left(\overrightarrow{\mathrm{v}}_{\varepsilon^{*}}\right)_{\left(n, \mathrm{f}_{\varepsilon}\right)}^{*}\right)_{\tilde{\beta}}^{\mathbb{T}_{s_{\varepsilon}}} \widehat{\mathrm{f}_{\varepsilon}}\right\rangle$ and $\left\langle\left(\overrightarrow{\mathrm{v}}_{w}\right)_{\tilde{\alpha}}^{\mathbb{T}_{s_{w}}} \widehat{\mathrm{f}_{w}}\right\rangle=\left\langle\left(\bar{\gamma}\left(\overrightarrow{\mathrm{v}}_{\epsilon^{*}}\right)_{\left(n, \mathrm{f}_{\epsilon}\right.}^{*}\right)_{\tilde{\alpha}}^{\mathbb{T}_{s_{\epsilon}}} \widehat{\mathrm{f}_{\epsilon}}\right\rangle$, by Lemma 10, uniqueness in Theorem 2 and because $\mathrm{U}_{0}^{*}, \mathrm{U}_{1}^{*}, \mathrm{U}_{2}^{*}, \mathrm{U}_{3}^{*}, \mathrm{U}_{4}^{*}, \mathrm{U}_{5}^{*}$ is a partition of $\{1, \ldots, r\}$,

$\Leftrightarrow \mathrm{U}_{1}=\mathrm{U}_{1}^{*}, \mathrm{U}_{4}=\mathrm{U}_{4}^{*}, \mathrm{U}_{5}=\mathrm{U}_{5}^{*}$, for each $u \in \mathrm{U}_{2}$ there exists $\varepsilon \in \mathrm{U}_{2}^{*}$, for each $w \in \mathrm{U}_{3}$ there exists $\epsilon \in \mathrm{U}_{3}^{*}$ such that $\left\langle\left(\overrightarrow{\mathrm{v}}_{u}\right)_{\tilde{\beta}}^{\mathbb{T}_{s_{u}}} \widehat{\mathrm{f}_{u}}\right\rangle=\left\langle\left(\bar{\gamma}\left(\overrightarrow{\mathrm{v}}_{\varepsilon}\right)_{\left(n, \mathrm{f}_{\varepsilon^{*}}\right)}^{*}\right)_{\tilde{\beta}}^{\mathbb{T}_{\varepsilon^{*}}} \widehat{\mathrm{f}_{\varepsilon^{*}}}\right\rangle$ and $\left\langle\left(\overrightarrow{\mathrm{v}}_{w}\right)_{\tilde{\alpha}}^{\mathbb{T}_{s_{w}}} \widehat{\mathrm{f}_{w}}\right\rangle=\left\langle\left(\bar{\gamma}\left(\overrightarrow{\mathrm{v}}_{\epsilon}\right)_{\left(n, \mathrm{f}_{\epsilon} *\right.}^{*}\right)_{\tilde{\alpha}}^{\mathbb{T}_{\epsilon^{*}}} \widehat{\mathrm{f}_{\epsilon^{*}}}\right\rangle$,

$\Leftrightarrow \mathrm{U}_{1}, \mathrm{U}_{2}, \mathrm{U}_{3}, \mathrm{U}_{4}, \mathrm{U}_{5}$ are self-reciprocal, for each $u \in \mathrm{U}_{2}$ there exists $\varepsilon \in \mathrm{U}_{2}$ such that $\mathrm{f}_{u}=\mathrm{f}_{\varepsilon}^{\circ *}$ and $\overrightarrow{\mathrm{v}}_{u}=\bar{\gamma}\left(\overrightarrow{\mathrm{v}}_{\varepsilon}\right)_{\left(n, \mathrm{f}_{\varepsilon^{*}}\right)}^{*}$, for each $w \in \mathrm{U}_{3}$ there exists $\epsilon \in \mathrm{U}_{3}$ such that $\mathrm{f}_{w}=\mathrm{f}_{\epsilon}^{\circ *}$ and $\overrightarrow{\mathrm{v}}_{w}=\bar{\gamma}\left(\overrightarrow{\mathrm{v}}_{\epsilon}\right)_{\left(n, \mathrm{f}_{\epsilon}^{*}\right)}^{*}$, by Lemma 5 ,

$\Leftrightarrow \mathrm{U}_{1}, \mathrm{U}_{2}, \mathrm{U}_{3}, \mathrm{U}_{4}, \mathrm{U}_{5}$ are self-reciprocal, for each $u \in \mathrm{U}_{2}, \overrightarrow{\mathrm{v}}_{u}=\bar{\gamma}\left(\overrightarrow{\mathrm{v}}_{u^{*}}\right)_{\left(n, \mathrm{f}_{u}\right)}^{*}$, and for each $w \in \mathrm{U}_{3}, \overrightarrow{\mathrm{v}}_{w}=\bar{\gamma}\left(\overrightarrow{\mathrm{v}}_{w^{*}}\right)_{\left(n, \mathrm{f}_{w}\right)}^{*}$,

$\Leftrightarrow \mathrm{U}_{1}, \mathrm{U}_{2}, \mathrm{U}_{3}, \mathrm{U}_{4}, \mathrm{U}_{5}$ are self-reciprocal and for each $u \in \mathrm{U}_{2} \cup \mathrm{U}_{3}, \overrightarrow{\mathrm{v}}_{u}=$ $\bar{\gamma}\left(\overrightarrow{\mathrm{v}}_{u^{*}}\right)_{\left(n, \mathrm{f}_{u}\right)}^{*}$.

$(2) \Leftrightarrow(3)$ It is easy to check.

Example 4. Let $\mathrm{R}=\mathrm{GF}(2)[\mathrm{X}, \mathrm{Y}] /\left\langle\mathrm{X}^{2}-\mathrm{Y}^{3}, \mathrm{XY}\right\rangle, \gamma, \tilde{\alpha}=\{\mathrm{x}, \mathrm{y}\}, \mathrm{f}_{1}, \mathrm{f}_{2}, \mathrm{f}_{3}$, $\mathrm{f}_{4}, \mathrm{f}_{5}$ as in Example 1, then $\bar{\gamma}=1,1^{*}=2,2^{*}=1,3^{*}=3,4^{*}=4,5^{*}=5$. Let $\mathrm{U}_{1}=\{4\}, \mathrm{U}_{2}=\{1,2\}, \mathrm{U}_{3}=\{3\}, \mathrm{U}_{4}=\{5\}, \mathrm{U}_{0}=\mathrm{U}_{5}=\emptyset, \overrightarrow{\mathrm{v}}_{1}=$ $(1, \mathrm{~T})$, over $\mathrm{GF}\left(2^{4}\right)=\mathrm{GF}(2)[\mathrm{T}] /\left\langle\mathrm{f}_{1}\right\rangle, \overrightarrow{\mathrm{v}}_{2}=\bar{\gamma}\left(\overrightarrow{\mathrm{v}}_{1}\right)_{\left(15, \mathrm{f}_{2}\right)}^{*}=\left(1, \mathrm{~T}^{3}+\mathrm{T}^{2}\right)$, over $\mathrm{GF}(2)[\mathrm{T}] /\left\langle\mathrm{f}_{2}\right\rangle$.

Since $\mathrm{T}^{3}+\mathrm{T}^{2} \in \mathrm{GF}(2)[\mathrm{T}] /\left\langle\mathrm{f}_{3}\right\rangle$ is a root of the irreducible polynomial $\mathrm{T}^{2}+\mathrm{T}+$ $1 \in \mathrm{GF}(2)[\mathrm{T}]$ then $\overrightarrow{\mathrm{v}}_{3}=\left(1, \mathrm{~T}^{3}+\mathrm{T}^{2}+1\right)$ over $\mathrm{GF}(2)[\mathrm{T}] /\left\langle\mathrm{f}_{3}\right\rangle$ has the property 
SELF DUAL, REVERSIBLE AND COMPLEMENTARY DUALS

CONSTACYCLIC CODES OVER FINITE LOCAL FROBENIUS NON-CHAIN

$\overrightarrow{\mathrm{v}}_{3}=\bar{\gamma}\left(\overrightarrow{\mathrm{v}}_{3}\right)_{\left(15, \mathrm{f}_{3}\right)}^{*}$, by Observation 2. Hence the $\gamma$-constacyclic code:

$$
\begin{gathered}
\mathrm{C}=\left\langle\mathfrak{m}^{3} \widehat{\prod_{u \in \mathrm{U}_{1}} \mathrm{f}_{u}}, \mathfrak{m} \widehat{\prod}_{u \in \mathrm{U}_{4}}, \widehat{\mathrm{f}_{u}}, \widehat{\mathrm{f}_{u}},\left(\overrightarrow{\mathrm{v}}_{u}\right)_{\tilde{\beta}}^{\mathbb{T}_{s_{u}}} \widehat{\mathrm{f}}_{u},\left(\overrightarrow{\mathrm{v}}_{w}\right)_{\tilde{\alpha}}^{\mathbb{T}_{s}} \widehat{\mathrm{f}}_{w}: u \in \mathrm{U}_{2}, w \in \mathrm{U}_{3}\right\rangle= \\
\left\langle\widehat{\mathrm{f}_{4}}, \mathfrak{m} \widehat{\mathrm{f}}_{5},[\mathrm{x}+\mathrm{Ty}] \widehat{\mathrm{f}_{1}},\left[\mathrm{x}+\left(\mathrm{T}^{3}+\mathrm{T}^{2}\right) \mathrm{y}\right] \widehat{\mathrm{f}_{2}},\left[\mathrm{x}+\left(\mathrm{T}^{3}+\mathrm{T}^{2}+1\right) \mathrm{y}\right] \widehat{\mathrm{f}_{3}}\right\rangle= \\
\left\langle\mathrm{f}_{1} \mathrm{f}_{2} \mathrm{f}_{3} \mathrm{f}_{5}, \mathfrak{m f}_{1} \mathrm{f}_{2} \mathrm{f}_{3} \mathrm{f}_{4},[\mathrm{x}+\mathrm{Ty}] \mathrm{f}_{2} \mathrm{f}_{3} \mathrm{f}_{4} \mathrm{f}_{5},\left[\mathrm{x}+\left(\mathrm{T}^{3}+\mathrm{T}^{2}\right) \mathrm{y}\right] \mathrm{f}_{1} \mathrm{f}_{3} \mathrm{f}_{4} \mathrm{f}_{5},\right. \\
\left.\left[\mathrm{x}+\left(\mathrm{T}^{3}+\mathrm{T}^{2}+1\right) \mathrm{y}\right] \mathrm{f}_{1} \mathrm{f}_{2} \mathrm{f}_{4} \mathrm{f}_{5}\right\rangle
\end{gathered}
$$

is reversible.

\section{Constacyclic codes with Complementary Duals over rings in $\mathfrak{F}_{5}^{4}$}

A linear code with complementary dual is defined to be a linear code $\mathrm{C}$ whose dual code satisfies $\mathrm{C} \cap \mathrm{C}^{\perp}=\langle 0\rangle$. In this Section the algebraic characterization of complementary dual $\gamma$-constacyclic codes over rings in the family $\mathfrak{F}_{5}^{4}$ is given, when $\gamma=\gamma^{-1}$.

Theorem 6. Let $(\mathrm{R}, \mathfrak{m}, \operatorname{GF}(q)) \in \mathfrak{F}_{5}^{4}, \gamma, \tilde{\alpha}=\left\{\alpha_{1}, \alpha_{2}\right\}, \tilde{\beta}=\left\{\alpha_{1}, \alpha_{2}^{2}\right\}, \mathbb{T}, \mathbb{T}_{s}$, $\mathrm{f}_{1}, \ldots, \mathrm{f}_{r}, s_{i}=\operatorname{deg}\left(\overline{\mathrm{f}}_{i}\right), \mathrm{C}$ as in Theorem 2 and

(1) $\mathrm{U}_{0}, \mathrm{U}_{1}, \mathrm{U}_{2}, \mathrm{U}_{3}, \mathrm{U}_{4}, \mathrm{U}_{5}$ the unique partition of $\{1, \ldots, r\}$, associated to $\mathrm{C}$,

(2) $\left\{\overrightarrow{\mathrm{v}}_{u}: u \in \mathrm{U}_{2} \cup \mathrm{U}_{3}\right\}$ the vectors such that the corresponding ideal, in $\mathrm{R}[\mathrm{T}] /\left\langle\mathrm{T}^{n}-\gamma\right\rangle$, of $\mathrm{C}$ is

$$
\left\langle\mathfrak{m}^{3} \widehat{\prod_{u \in \mathrm{U}_{1}} \mathrm{f}_{u}}, \mathfrak{m} \widehat{\prod_{u \in \mathrm{U}_{4}} \mathrm{f}_{u}}, \widehat{\prod_{u \in \mathrm{U}_{5}} \mathrm{f}_{u}},\left(\overrightarrow{\mathrm{v}}_{u}\right)_{\tilde{\beta}}^{\mathbb{T}_{s_{u}}} \widehat{\mathrm{f}}_{u},\left(\overrightarrow{\mathrm{v}}_{w}\right)_{\tilde{\alpha}}^{\mathbb{T}_{s}} \widehat{\mathrm{f}}_{w}: u \in \mathrm{U}_{2}, w \in \mathrm{U}_{3}\right\rangle .
$$

The following conditions are equivalent:

(1) $\mathrm{C}$ is a Complementary dual code;

(2) $\mathrm{U}_{0}$ and $\mathrm{U}_{5}$ are self-reciprocal and $\mathrm{U}_{i}=\emptyset$, for $i \in\{1,2,3,4\}$;

(3) $\mathrm{U}_{5}$ is self-reciprocal and $\mathrm{U}_{i}=\emptyset$, for $i \in\{1,2,3,4\}$.

In this case $\mathrm{C}=\left\langle\widehat{\prod}_{u \in \mathrm{U}_{5}} \mathrm{f}_{u}\right\rangle$ and $\mathrm{C}^{\perp}=\left\langle\widehat{\prod}_{u \in \mathrm{U}_{0}} \mathrm{f}_{u}\right\rangle$. 
SELF DUAL, REVERSIBLE AND COMPLEMENTARY DUALS

CONSTACYCLIC CODES OVER FINITE LOCAL FROBENIUS NON-CHAIN

RINGS OF LENGTH 5 AND NILPOTENCY INDEX 4

Proof: First recall the following facts: The intersection of two non zero ideals of $\mathrm{R}$ is not zero; if $\mathrm{U}=\left\{u_{1}, \ldots, u_{k}\right\} \subseteq\{1, r\}$, the ideal generated by $\widehat{\prod_{u \in \mathrm{U}} \mathrm{f}_{u}}$ in $\mathrm{R}[\mathrm{T}] /\left\langle\mathrm{T}^{n}-\gamma\right\rangle$ is identified with $\langle\overrightarrow{0}\rangle \oplus \mathrm{R}[\mathrm{T}] /\left\langle\mathrm{f}_{u_{1}}\right\rangle \oplus\langle\overrightarrow{0}\rangle \oplus \ldots \oplus\langle\overrightarrow{0}\rangle \oplus$ $\mathrm{R}[\mathrm{T}] /\left\langle\mathrm{f}_{u_{k}}\right\rangle \oplus\langle\overrightarrow{0}\rangle$; the rings $\mathrm{R}[\mathrm{T}] /\left\langle\mathrm{f}_{i}\right\rangle$ are Frobenius, $i \in\{1, \ldots, r\}$.

Now, from Theorem 3,

$$
\begin{gathered}
\mathrm{C}^{\perp}=\left\langle\mathfrak{m}^{3} \widehat{\prod_{u \in \mathrm{U}_{4}^{*}} \mathrm{f}_{u}}, \mathfrak{m} \widehat{\prod_{u \in \mathrm{U}_{1}^{*}} \mathrm{f}_{u}}, \widehat{\prod_{u \in \mathrm{U}_{0}^{*}} \mathrm{f}_{u}},\left(\bar{\gamma}\left(\overrightarrow{\mathrm{v}}_{w^{*}}^{\perp}\right)_{\left(n, \mathrm{f}_{w}\right)}^{*}\right)_{\tilde{\beta}}^{\mathbb{T}_{s}} \widehat{\mathrm{f}_{w}},\right. \\
\left.\left(\bar{\gamma}\left(\overrightarrow{\mathrm{v}}_{u^{*}}^{\perp}\right)_{\left(n, \mathrm{f}_{u}\right)}^{*}\right)_{\tilde{\alpha}}^{\mathbb{T}_{s_{u}}} \widehat{\mathrm{f}_{u}}: u \in \mathrm{U}_{2}^{*}, w \in \mathrm{U}_{3}^{*}\right\rangle .
\end{gathered}
$$

Then $\mathrm{C} \cap \mathrm{C}^{\perp}=\langle 0\rangle$

$\Leftrightarrow\left[\mathrm{U}_{0}^{*} \cup \mathrm{U}_{1}^{*} \cup \mathrm{U}_{2}^{*} \cup \mathrm{U}_{3}^{*} \cup \mathrm{U}_{4}^{*}\right] \cap\left[\mathrm{U}_{1} \cup \mathrm{U}_{2} \cup \mathrm{U}_{3} \cup \mathrm{U}_{4} \cup \mathrm{U}_{5}\right]=\emptyset$

$\Leftrightarrow \mathrm{U}_{0}^{*} \cup \mathrm{U}_{1}^{*} \cup \mathrm{U}_{2}^{*} \cup \mathrm{U}_{3}^{*} \cup \mathrm{U}_{4}^{*}=\{1, \ldots, r\} \backslash \mathrm{U}_{5}^{*} \subseteq \mathrm{U}_{0}$

$\Leftrightarrow \mathrm{U}_{0}^{*}=\mathrm{U}_{0}, \mathrm{U}_{1}^{*}=\mathrm{U}_{2}^{*}=\mathrm{U}_{3}^{*}=\mathrm{U}_{4}^{*}=\emptyset$, because $\left|\mathrm{U}_{0}^{*}\right|=\left|\mathrm{U}_{0}\right|$

$\Leftrightarrow \mathrm{U}_{0}$ and $\mathrm{U}_{5}$ are self-reciprocal and $\mathrm{U}_{1}^{*}=\mathrm{U}_{2}^{*}=\mathrm{U}_{3}^{*}=\mathrm{U}_{4}^{*}=\emptyset$

$\Leftrightarrow \mathrm{U}_{5}$ is self-reciprocal and $\mathrm{U}_{1}^{*}=\mathrm{U}_{2}^{*}=\mathrm{U}_{3}^{*}=\mathrm{U}_{4}^{*}=\emptyset$.

Example 5. Let $\mathrm{R}=\mathrm{GF}(2)[\mathrm{X}, \mathrm{Y}] /\left\langle\mathrm{X}^{2}-\mathrm{Y}^{3}, \mathrm{XY}\right\rangle, \gamma, \tilde{\alpha}=\{\mathrm{x}, \mathrm{y}\}, \mathrm{f}_{1}, \mathrm{f}_{2}, \mathrm{f}_{3}, \mathrm{f}_{4}$, $\mathrm{f}_{5}$ as in Example 1, then $1^{*}=2,2^{*}=1,3^{*}=3,4^{*}=4,5^{*}=5$ and the selfreciprocal subsets of $\{1,2,3,4,5\}$ are $\{1,2\},\{1,2,3\},\{1,2,3,4\},\{1,2,3,4,5\}$, $\{1,2,4\},\{1,2,4,5\},\{1,2,5\},\{3\},\{3,4\},\{3,4,5\}\{4\},\{4,5\},\{5\}$ and $\emptyset$.

The Complementary $\gamma$-constacyclic code over $\mathrm{R}$ of length 15 and their duals are the following:

(1) $\mathrm{C}=\left\langle\widehat{\mathrm{f}_{1} \mathrm{f}_{2}}\right\rangle=\left\langle\mathrm{f}_{3} \mathrm{f}_{4} \mathrm{f}_{5}\right\rangle$ and its dual is $\mathrm{C}^{\perp}=\left\langle\widehat{\mathrm{f}_{3} \mathrm{f}_{4} \mathrm{f}_{5}}\right\rangle=\left\langle\mathrm{f}_{1} \mathrm{f}_{2}\right\rangle$;

(2) $\mathrm{C}=\left\langle\widehat{\mathrm{f}_{1} \mathrm{f}_{2} \mathrm{f}_{3}}\right\rangle=\left\langle\mathrm{f}_{4} \mathrm{f}_{5}\right\rangle$ and its dual is $\mathrm{C}^{\perp}=\left\langle\widehat{\mathrm{f}_{4} \mathrm{f}_{5}}\right\rangle=\left\langle\mathrm{f}_{1} \mathrm{f}_{2} \mathrm{f}_{3}\right\rangle$;

(3) $\mathrm{C}=\left\langle\widehat{\mathrm{f}_{1} \mathrm{f}_{2} \mathrm{f}_{3} \mathrm{f}_{4}}\right\rangle=\left\langle\mathrm{f}_{5}\right\rangle$ and its dual is $\mathrm{C}^{\perp}=\left\langle\widehat{\mathrm{f}_{5}}\right\rangle=\left\langle\mathrm{f}_{1} \mathrm{f}_{2} \mathrm{f}_{3} \mathrm{f}_{4}\right\rangle$;

(4) $\mathrm{C}=\left\langle\mathrm{f}_{1} \widehat{\mathrm{f}_{2} \mathrm{f}_{3} \mathrm{f}_{4}} \mathrm{f}_{5}\right\rangle=\langle 1\rangle$ and its dual is $\mathrm{C}^{\perp}=\langle 0\rangle$;

(5) $\mathrm{C}=\left\langle\widehat{\mathrm{f}_{1} \mathrm{f}_{2} \mathrm{f}_{4}}\right\rangle=\left\langle\mathrm{f}_{3} \mathrm{f}_{5}\right\rangle$ and its dual is $\mathrm{C}^{\perp}=\left\langle\widehat{\mathrm{f}_{3} \mathrm{f}_{5}}\right\rangle=\left\langle\mathrm{f}_{1} \mathrm{f}_{2} \mathrm{f}_{4}\right\rangle$;

(6) $\mathrm{C}=\left\langle\widehat{\mathrm{f}_{1} \mathrm{f}_{2} \mathrm{f}_{4}} \mathrm{f}_{5}\right\rangle=\left\langle\mathrm{f}_{3}\right\rangle$ and its dual is $\mathrm{C}^{\perp}=\left\langle\widehat{\mathrm{f}_{3}}\right\rangle=\left\langle\mathrm{f}_{1} \mathrm{f}_{2} \mathrm{f}_{4} \mathrm{f}_{5}\right\rangle$;

(7) $\mathrm{C}=\left\langle\widehat{\mathrm{f}_{1} \mathrm{f}_{2} \mathrm{f}_{5}}\right\rangle=\left\langle\mathrm{f}_{3} \mathrm{f}_{4}\right\rangle$ and its dual is $\mathrm{C}^{\perp}=\left\langle\widehat{\mathrm{f}_{3} \mathrm{f}_{4}}\right\rangle=\left\langle\mathrm{f}_{1} \mathrm{f}_{2} \mathrm{f}_{5}\right\rangle$;

(8) $\mathrm{C}=\left\langle\widehat{\mathrm{f}_{3}}\right\rangle=\left\langle\mathrm{f}_{1} \mathrm{f}_{2} \mathrm{f}_{4} \mathrm{f}_{5}\right\rangle$ and its dual is $\mathrm{C}^{\perp}=\left\langle\widehat{\mathrm{f}_{1} \mathrm{f}_{2} \mathrm{f}_{4} \mathrm{f}_{5}}\right\rangle=\left\langle\mathrm{f}_{3}\right\rangle$;

(9) $\mathrm{C}=\left\langle\widehat{\mathrm{f}_{3} \mathrm{f}_{4}}\right\rangle=\left\langle\mathrm{f}_{1} \mathrm{f}_{2} \mathrm{f}_{5}\right\rangle$ and its dual is $\mathrm{C}^{\perp}=\left\langle\widehat{\mathrm{f}_{1} \mathrm{f}_{2} \mathrm{f}_{5}}\right\rangle=\left\langle\mathrm{f}_{3} \mathrm{f}_{4}\right\rangle$;

(10) $\mathrm{C}=\left\langle\widehat{\mathrm{f}_{3} \mathrm{f}_{4} \mathrm{f}_{5}}\right\rangle=\left\langle\mathrm{f}_{1} \mathrm{f}_{2}\right\rangle$ and its dual is $\mathrm{C}^{\perp}=\left\langle\widehat{\mathrm{f}_{1} \mathrm{f}_{2}}\right\rangle=\left\langle\mathrm{f}_{3} \mathrm{f}_{4} \mathrm{f}_{5}\right\rangle$; 
SELF DUAL, REVERSIBLE AND COMPLEMENTARY DUALS

CONSTACYCLIC CODES OVER FINITE LOCAL FROBENIUS NON-CHAIN

RINGS OF LENGTH 5 AND NILPOTENCY INDEX 4

(11) $\mathrm{C}=\left\langle\widehat{\mathrm{f}_{4}}\right\rangle=\left\langle\mathrm{f}_{1} \mathrm{f}_{2} \mathrm{f}_{3} \mathrm{f}_{5}\right\rangle$ and its dual is $\mathrm{C}^{\perp}=\left\langle\widehat{\mathrm{f}_{1} \mathrm{f}_{2} \mathrm{f}_{3} \mathrm{f}_{5}}\right\rangle=\left\langle\mathrm{f}_{4}\right\rangle$;

(12) $\mathrm{C}=\left\langle\widehat{\mathrm{f}_{4} \mathrm{f}_{5}}\right\rangle=\left\langle\mathrm{f}_{1} \mathrm{f}_{2} \mathrm{f}_{3}\right\rangle$ and its dual is $\mathrm{C}^{\perp}=\left\langle\widehat{\mathrm{f}_{1} \mathrm{f}_{2} \mathrm{f}_{3}}\right\rangle=\left\langle\mathrm{f}_{4} \mathrm{f}_{5}\right\rangle$;

(13) $\mathrm{C}=\left\langle\widehat{\mathrm{f}}_{5}\right\rangle=\left\langle\mathrm{f}_{1} \mathrm{f}_{2} \mathrm{f}_{3} \mathrm{f}_{4}\right\rangle$ and its dual is $\mathrm{C}^{\perp}=\left\langle\widehat{\mathrm{f}_{1} \mathrm{f}_{2} \mathrm{f}_{3} \mathrm{f}_{4}}\right\rangle=\left\langle\mathrm{f}_{5}\right\rangle$;

(14) $\mathrm{C}=\langle 0\rangle$ and its dual is $\mathrm{C}^{\perp}=\langle 1\rangle$.

\section{Acknowledgements}

The authors would like to thank the referees for their valuable observations and comments.

\section{References}

[1] A. Bayram, E. S. Oztas, I. Siap, Codes over $\mathbb{F}_{4}+v \mathbb{F}_{4}$ and some DNA applications, Des. Codes Cryptogr. 80 379-393 (2016)

[2] C. A. Castillo-Guillén, C. Rentería-Márquez, H. Tapia-Recillas, Constacyclic codes over finite local Frobenius non-chain rings with nilpotency index 3, Finite Fields Appl. 43 1-21 (2017)

[3] C. A. Castillo-Guillén, C. Rentería-Márquez, H. Tapia-Recillas, Duals of constacyclic codes over finite local Frobenius non-chain rings of length 4, Discrete Math. 341 919-933 (2018)

[4] C. A. Castillo-Guillén, C. Rentería-Márquez, E. Sarmiento-Rosales, H. Tapia-Recillas, R. H. Villareal, The dual of a constacyclic code, self dual and reversible constacyclic codes and constacyclic codes with complementary duals over finite local Frobenius non-chain rings of nilpotency index 3, Discrete Math. 342 2283-2296 (2019)

[5] C. A. Castillo-Guillén, C. Rentería-Márquez, Constacyclic codes over finite local Frobenius non-chain rings of length 5 and nilpotency index 4, An. Stiint. Univ. Ovidius Constanta Ser. Mat. (to appear)

[6] H. Q. Dinh, S. R. López-Permouth, Cyclic and Negacyclic Codes Over Finite Chain Rings, IEEE Trans. Inf. Theory 50 1728-1744 (2004)

[7] A. R. Hammons, P. V. Kumar, A. R. Calderbank, N. J. A. Sloane, P. Solé, The $\mathbb{Z}_{4}$-linearity of Kerdock, Preparata, Goethals, and related codes, IEEE Trans. Inf. Theory 40 301-319 (1994) 
SELF DUAL, REVERSIBLE AND COMPLEMENTARY DUALS

CONSTACYCLIC CODES OVER FINITE LOCAL FROBENIUS NON-CHAIN

RINGS OF LENGTH 5 AND NILPOTENCY INDEX 4

[8] J. Kaur, R. Sehmi, S. Dutt, Reversible complement cyclic codes over Galois rings with applications to DNA codes, Discrete Appl. Math. 280 162-170 (2020)

[9] X. Liu, H. Liu, LCD codes over finite chain rings, Finite Fields Appl. 34 $1-19(2015)$

[10] J. L. Massey, Reversible codes, Inf. control 7 369-380 (1964)

[11] J. L. Massey, Linear codes with complementary duals, Discrete Math. 106/107 337-342 (1992)

[12] B. R. McDonald, Finite Rings with Identity, Marcel Dekker, New York, 1974

[13] N. Sendrier, Linear codes with complementary dual meet the GilbertVarshamov bound, Discrete Math. 285 345-347 (2004)

[14] M. Shi, D. Huang, L. Sok, P. Solé, Double circulant LCD codes over $\mathbb{Z}_{4}$, Finite Fields Appl. 58 133-144 (2019)

[15] J. Wood, Duality for modules over finite rings and applications to coding theory. Amer. J. Math. 121 555-575 (1999)

[16] X. Yang and J. L. Massey, The condition for a cyclic code to have a complementary dual, Discrete Math. 126 391-393 (1994)

[17] B. Yildiz, I. Siap, Cyclic codes over $\mathbb{F}_{2}[u] /\left\langle u^{4}-1\right\rangle$ and applications to DNA codes, Comput. Math. Appl. 63 1169-1176 (2012)

C. A. Castillo-Guillén

Department of Mathematics,

Universidades para el Bienestar Benito Juárez García,

Tláhuac Ave 6391, 13360, México City, México

Email:carlos_53@hotmail.com

C. Álvarez-García

Department of Mathematics,

Universidad Autónoma Metropolitana-I,

San Rafael Atlixco Ave 186, 09340, México City, México

Email:cainalvarez@hotmail.com 